\title{
Guide rapide des indicateurs de l'éducation \\ pour l'ODD 4
}

\section{逢哜}

Fir

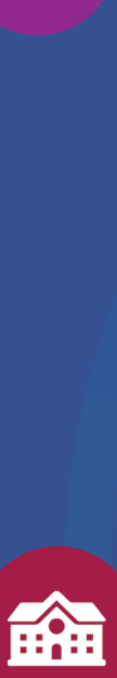

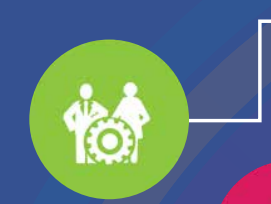
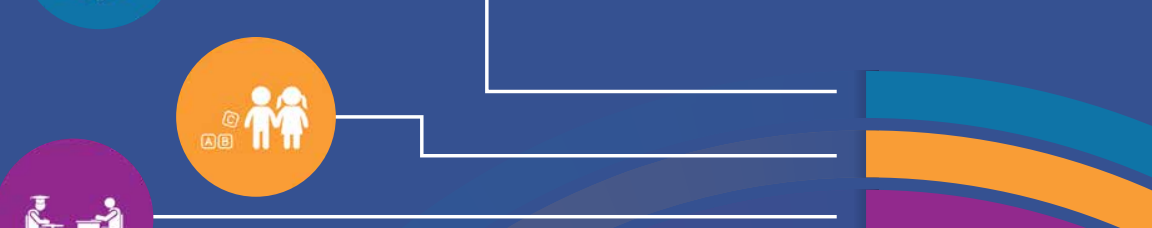

怔

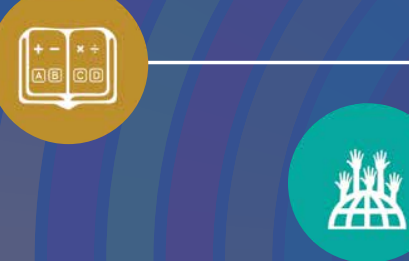

\section{(3)}

\begin{tabular}{|l|}
\hline त्र \\
\hline
\end{tabular}

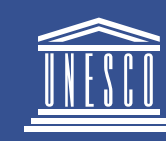

Allli, msstrut

Organisation 



\section{Guide rapide des indicateurs de l'éducation pour l'ODD 4}
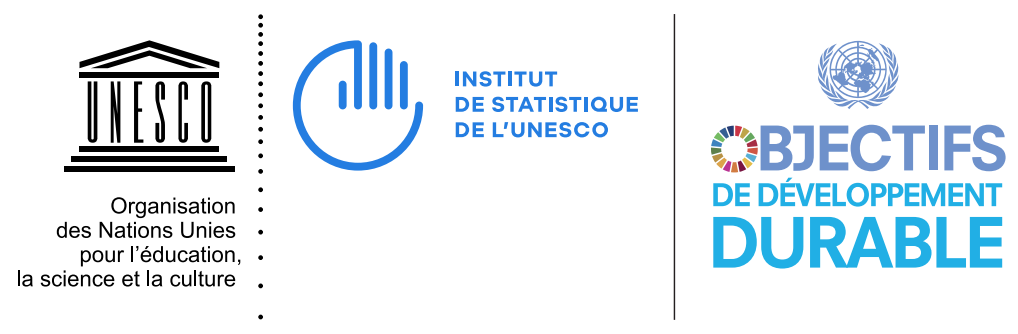


\section{UNESCO}

L'Acte constitutif de l'Organisation des Nations Unies pour l'éducation, la science et la culture (UNESCO) a été adopté par 20 pays lors de la Conférence de Londres en novembre 1945 et est entré en vigueur le 4 novembre 1946. L'UNESCO compte actuellement 195 États membres et 11 membres associés.

L'objectif premier de l'UNESCO est de contribuer au maintien de la paix et de la sécurité dans le monde en resserrant, par l'éducation, la science et la culture, la collaboration entre nations afin d'assurer le respect universel de la justice, de la loi, des droits de l'homme et des libertés fondamentales pour tous, sans distinction de race, de sexe, de langue ou de religion, que la Charte des Nations Unies reconnaît à tous les peuples.

L'UNESCO a cinq fonctions principales, inscrites dans son mandat : 1) des études prospectives sur l'éducation, la science, la culture et la communication dans le monde de demain ; 2) le progrès, le transfert et le partage des connaissances par des activités de recherche, de formation et d'enseignement ; 3) des actions normatives en vue de la préparation et de l'adoption d'instruments internationaux et de recommandations réglementaires ; 4) l'expertise par le biais de la coopération technique avec les États membres, en faveur de leurs projets et politiques de développement ; 5) l'échange d'informations spécialisées.

\section{Institut de statistique de I'UNESCO}

L'Institut de statistique de l'UNESCO (ISU) est le bureau de statistique de l'UNESCO, chargé de rassembler, pour le compte des Nations Unies, des statistiques mondiales dans les domaines de l'éducation, de la science, la technologie et l'innovation, et de la culture et la communication.

L'ISU a été créé en 1999 avec pour mission d'améliorer le programme statistique de l'UNESCO et d'élaborer et mettre à disposition des statistiques actualisées, précises et pertinentes pour les politiques, comme l'exige le contexte social, politique et économique actuel de plus en plus complexe et changeant.

Publié en 2018 par :

Institut de statistique de l'UNESCO

C.P. 6128, Succursale Centre-Ville

Montréal, Québec H3C 3J7

Canada

Téléphone : (1 514) 343-6880

Courriel : uis.publications@unesco.org

http://www.uis.unesco.org

Réf : UIS/2018/ED/TD/4

(c) UNESCO-UIS 2018

Cette publication est disponible en accès libre sous la licence Attribution-ShareAlike 3.0 IGO (CC-BY-SA 3.0 IGO) (http://creativecommons.org/licenses/by-sa/3.0/igo/). En utilisant le contenu de cette publication, les utilisateurs acceptent de se conformer aux conditions d'utilisation du service d'archive des publications en accès libre de l'UNESCO (http://fr.unesco.org/ open-access/terms-use-ccbysa-fr).

Les appellations employées dans cette publication et la présentation des données qui y figurent n'impliquent de la part de I'UNESCO aucune prise de position quant au statut juridique de tout pays, territoire, ville ou zone ou de leurs autorités, ni quant au tracé de leurs frontières ou limites.

Les idées et opinions exprimées dans cette publication sont celles des auteurs. Elles ne représentent pas nécessairement les vues de l'UNESCO et n'engagent à rien l'Organisation. 


\section{Qu'est-ce que ce guide?}

Ce guide sert de référence rapide sur la manière de suivre les progrès accomplis vers la réalisation de l'Objectif de développement durable 4 (ODD 4) sur une éducation de qualité. II fournit les explications essentielles sur les cibles de l'ODD 4, leurs indicateurs, le mode d'élaboration des indicateurs et où trouver les données nécessaires à ces indicateurs.

\section{Table des matières}

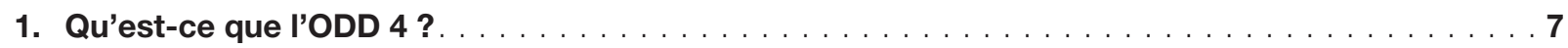

2. Quels sont les outils existants pour mesurer les progrès accomplis vers la réalisation

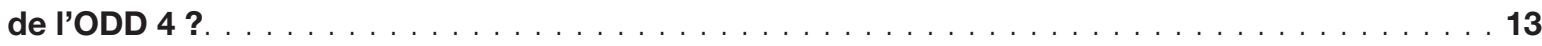

3. Quel est le rôle de l'ISU dans le suivi de l'ODD 4 ? $\ldots \ldots \ldots \ldots \ldots \ldots \ldots \ldots \ldots \ldots \ldots$

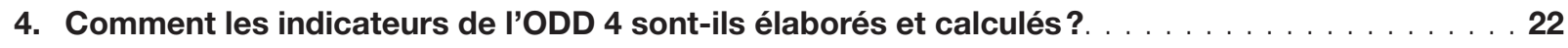

4.1 Enseignement primaire et secondaire gratuit, sur un pied d'égalité et de qualité $\ldots \ldots \ldots \ldots .24$

4.2 Activités de développement et de soins de la petite enfance et éducation préscolaire de qualité . . .27

4.3 Enseignement et formation techniques et professionnels (EFTP) et enseignement supérieur

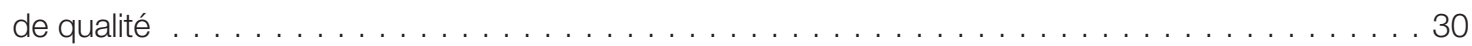

4.4 Compétences techniques et professionnelles $\ldots \ldots \ldots \ldots \ldots \ldots \ldots \ldots \ldots \ldots \ldots \ldots \ldots$

4.5 Égalité d'accès des personnes vulnérables à tous les niveaux d'enseignement et de

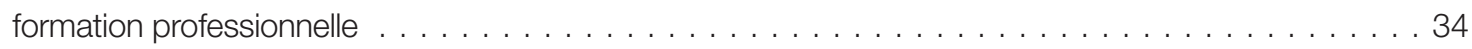

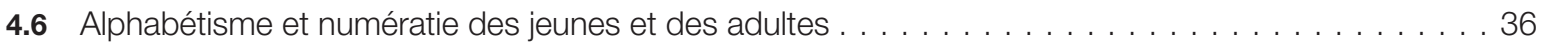

4.7 Connaissances et compétences nécessaires pour promouvoir le développement durable. . . . . . 38

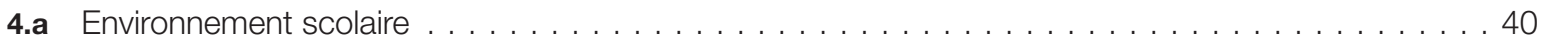

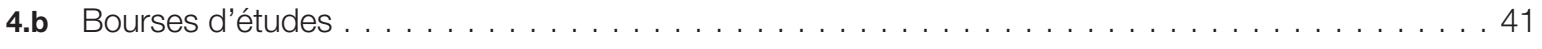

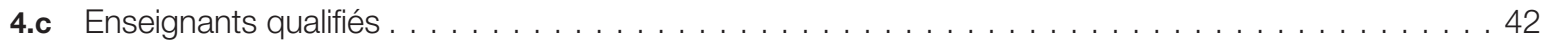

Annexe. Cibles et indicateurs de l'Objectif de développement durable 4 sur l'éducation . . . . . . 43

Références . . . . . . . . . . . 46 


\section{Sigles}

\begin{tabular}{|c|c|}
\hline APD & Aide publique au développement \\
\hline CAD & Comité d'aide au développement \\
\hline CEA & Catalogue sur les évaluations de l'apprentissage \\
\hline CEPALC & Commission économique pour l'Amérique latine et les Caraïbes \\
\hline CITE & Classification internationale type de l'éducation \\
\hline CNE & Compte national de l'éducation \\
\hline CONFEMEN & Conférence des ministres de l'Éducation des États et gouvernements de la Francophonie \\
\hline DSNU & Division de statistique des Nations Unies \\
\hline ECM & Éducation à la citoyenneté mondiale \\
\hline EDD & Éducation au développement durable \\
\hline EDS & Enquête démographique et de santé \\
\hline EEA & Enquête sur l'éducation des adultes \\
\hline EFTP & Enseignement et formation techniques et professionnels \\
\hline EPT & Éducation pour tous \\
\hline ETVA & Enquête sur la transition vers la vie active \\
\hline FPHN & Forum politique de haut niveau pour le développement durable \\
\hline GAML & Global Alliance to Monitor Learning (Alliance mondiale pour le suivi de l'apprentissage) \\
\hline GCT & Groupe de coopération technique sur les indicateurs de l’ODD 4-Éducation 2030 \\
\hline GEMR & Global Education Monitoring Report (Rapport mondial de suivi sur l'éducation) \\
\hline GIA-IIE & Groupe inter-agences sur les indicateurs de l'inégalité dans l'éducation \\
\hline GIAE-ODD & Groupe inter-agences et d'experts sur les indicateurs des Objectifs de développement durable \\
\hline IDPE & Indice de développement de la petite enfance \\
\hline IEA & $\begin{array}{l}\text { International Association for the Evaluation of Educational Achievement (Association internationale pour } \\
\text { l'évaluation du rendement scolaire) }\end{array}$ \\
\hline IIPE & Institut international de planification de l'éducation de l’UNESCO \\
\hline IPS & Indice de parité entre les sexes \\
\hline IPUMS & Integrated Public Use Microdata Series (Séries de microdonnées intégrées à usage public) \\
\hline ISU & Institut de statistique de l’UNESCO \\
\hline LAMP & Literacy Assessment and Monitoring Programme (Programme d'évaluation et de suivi de l'alphabétisation) \\
\hline LaNA & Literacy and Numeracy Assessment (Évaluation des aptitudes à la lecture, à l'écriture et au calcul) \\
\hline LLECE & $\begin{array}{l}\text { Latin American Laboratory for the Assessment of the Quality of Education (Laboratoire latino-américain } \\
\text { d'évaluation de la qualité de l'éducation) }\end{array}$ \\
\hline MELQO & $\begin{array}{l}\text { Measuring Early Learning Quality and Outcomes (Mesure de la qualité et des acquis d'apprentissage } \\
\text { préscolaire) }\end{array}$ \\
\hline MICS & Multiple Indicator Cluster Surveys (Enquêtes par grappes à indicateurs multiples) \\
\hline NEQMAP & $\begin{array}{l}\text { Network on Education Quality Monitoring in the Asia-Pacific (Réseau sur le suivi de la qualité de l'éducation } \\
\text { en Asie-Pacifique) }\end{array}$ \\
\hline OCDE & Organisation de coopération et de développement économiques \\
\hline ODD & Objectifs de développement durable \\
\hline OMD & Objectifs du Millénaire pour le développement \\
\hline ONG & rganisation non gouvernementale \\
\hline
\end{tabular}




\begin{tabular}{|c|c|}
\hline OOSCI & Out-of-School Children Initiative (Initiative mondiale en faveur des enfants non scolarisés) \\
\hline PASEC & Programme d'analyse des systèmes éducatifs de la CONFEMEN \\
\hline PIAAC & $\begin{array}{l}\text { Programme for the International Assessment of Adult Competencies (Programme pour l'évaluation } \\
\text { internationale des compétences des adultes) }\end{array}$ \\
\hline PILNA & $\begin{array}{l}\text { Pacific Islands Literacy and Numeracy Assessment (Évaluation des aptitudes à la lecture, à l'écriture et au } \\
\text { calcul dans les îles du Pacifique) }\end{array}$ \\
\hline PIRLS & Programme international de recherche en lecture scolaire \\
\hline PISA & Programme international pour le suivi des acquis des élèves \\
\hline PME & Partenariat mondial pour l'éducation \\
\hline PRIDI & $\begin{array}{l}\text { Programa Regional de Indicadores de Desarrollo Infantil (Programme régional des indicateurs pour le } \\
\text { développement de l'enfant) }\end{array}$ \\
\hline SACMEQ & $\begin{array}{l}\text { Southern and Eastern Africa Consortium for Monitoring Educational Quality (Consortium de l'Afrique australe } \\
\text { et orientale pour le pilotage de la qualité de l'éducation) }\end{array}$ \\
\hline SEAMEO & $\begin{array}{l}\text { Southeast Asia Ministers of Education Organization (Organisation des ministres de l'Éducation d'Asie du } \\
\text { Sud-Est) }\end{array}$ \\
\hline SEA-PLM & $\begin{array}{l}\text { Southeast Asia Primary Learning Metrics (Métriques de l'apprentissage à l'école primaire en Asie du Sud- } \\
\text { Est) }\end{array}$ \\
\hline SIGE & Système d'information pour la gestion de l'éducation \\
\hline SLS & Short Literacy Survey (Courte enquête sur l'alphabétisme) \\
\hline SMC & Seuil minimal de compétence \\
\hline STEP & Skills Toward Employment and Productivity (Compétences pour l'employabilité et la productivité) \\
\hline TERCE & Tercer Estudio Regional Comparativo y Explicativo (Troisième étude régionale comparative et explicative) \\
\hline TIC & Technologies de l'information et de la communication \\
\hline TIMSS & $\begin{array}{l}\text { Trends in International Mathematics and Science Study (Tendances de l'enquête internationale sur les } \\
\text { mathématiques et les sciences) }\end{array}$ \\
\hline UIT & Union internationale des télécommunications \\
\hline UNESCO & $\begin{array}{l}\text { United Nations Organization for Education, Science and Culture (Organisation des Nations Unies pour } \\
\text { l'éducation, la science et la culture) }\end{array}$ \\
\hline UNICEF & United Nations Children's Fund (Fonds des Nations Unies pour l'enfance) \\
\hline USAID & $\begin{array}{l}\text { United States Agency for International Development (Agence des États-Unis pour le développement } \\
\text { international) }\end{array}$ \\
\hline
\end{tabular}





\section{Qu'est-ce que l'ODD 4 ?}

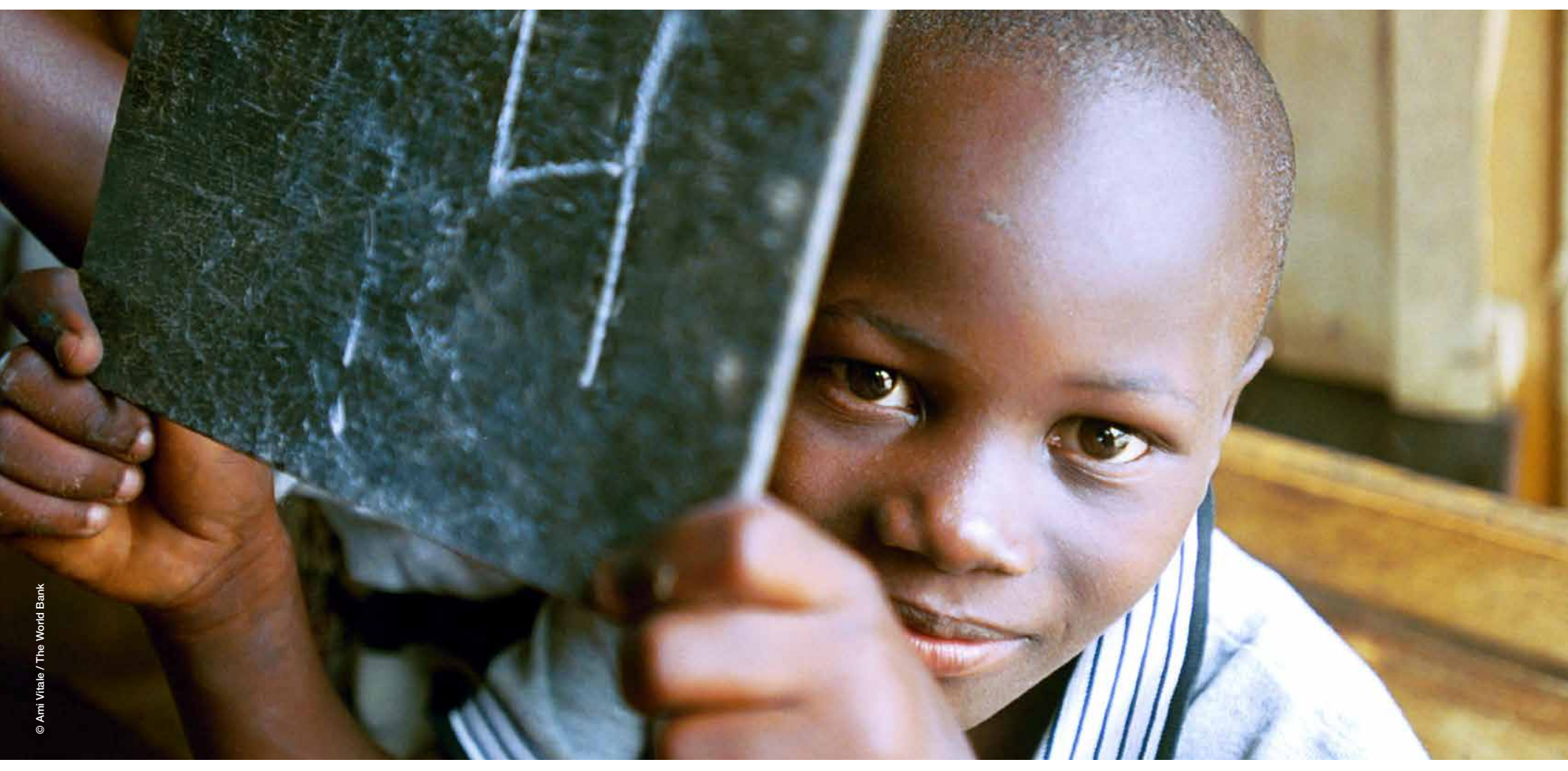

Les chefs d'État et de gouvernement, les représentants de haut niveau des Nations Unies et la société civile se sont réunis en 2015 lors de la 70 session de l'Assemblée générale des Nations Unies et ont adopté l'Agenda de développement durable 2030. Ce programme ambitieux «des peuples, par les peuples et pour les peuples » a été conçu avec la participation active de I'UNESCO. L'éducation joue un rôle central dans l'Agenda 2030 qui contient un objectif spécifique pour l'éducation et des cibles relatives à l'éducation dans 7 des 17 autres

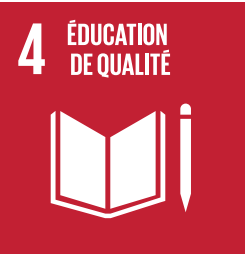
Objectifs de développement durable (ODD). L'ODD 4 vise à «assurer l'accès de tous à une éducation de qualité, sur un pied d'égalité, et promouvoir les possibilités d'apprentissage tout au long de la vie" d'ici 2030. L'objectif comporte dix cibles pour guider les pays sur le chemin de la transformation vers un programme d'éducation durable.

Qu'est-ce qu'une cible? Une cible est un objectif spécifique, mesurable, qui contribuera à réaliser un ou plusieurs objectifs. L'ODD 4 a dix cibles couvrant de nombreux aspects différents de l'éducation, parmi lesquelles sept cibles sont des résultats escomptés et trois des moyens de réaliser ces résultats.

Comment les progrès de l'ODD 4 peuvent-ils être mesurés? L'ODD 4 couvre un spectre plus large que ses prédécesseurs : l'Objectif du Millénaire pour le développement (OMD) 2 : «Assurer l'éducation primaire pour tous", et l'OMD 3 : «Promouvoir l'égalité des sexes et l'autonomisation des femmes». Sur le plan statistique, le cadre des OMD était fondé sur un ensemble d'indicateurs précis et mesurables et il a contribué à améliorer les capacités nationales de suivi de nombreux pays en développement. En revanche, les cibles de l'ODD 4 sont 
plus complexes et contiennent plusieurs concepts qui n'ont jamais été mesurés auparavant au niveau mondial, ce qui pose de nouveaux défis pour élaborer les indicateurs permettant de suivre les progrès accomplis vers la réalisation de l'ODD 4.

Qu'est-ce qu'un indicateur? Les indicateurs sont des marqueurs de changement ou de continuité qui nous permettent de mesurer, par exemple, le chemin parcouru en matière de développement. Dans le cadre des ODD, ils décrivent la façon dont une unité donnée (élève, école, pays ou région) progresse en relation avec une cible spécifique. Les indicateurs de l'ODD 4 revêtent de multiples facettes et beaucoup nécessitent d'élaborer de nouvelles méthodologies, définitions et méthodes de calcul, ainsi que de modifier considérablement les systèmes nationaux de communication des données, tant au plan national qu'international.

Quelle est la différence entre une cible et un indicateur? Si vous avez une cible personnelle, disons terminer un marathon cette année, un indicateur possible pour suivre vos progrès est la distance que vous pouvez courir chaque jour pendant votre préparation. Réaliser des distances plus longues indique que vous vous rapprochez de votre cible.

Les cibles de l'éducation sont toutefois beaucoup plus complexes que courir $42 \mathrm{~km}$ et souvent plus épuisantes. Le processus pour apprendre à jouer d'un nouvel instrument de musique offre une meilleure analogie. Vous pourriez vous fixer comme cible personnelle d'apprendre à jouer de la guitare cette année. Mais quel niveau de maîtrise devrez-vous avoir pour dire que vous savez en jouer? Vous pourriez définir une cible plus spécifique comme jouer une chanson particulière ou apprendre à jouer un certain nombre d'accords. De plus, choisir un ou plusieurs indicateurs pour suivre vos progrès peut être aussi complexe que de définir la cible : Le nombre d'heures consacrées à apprendre? Le nombre d'accords ou de parties d'une chanson que vous savez jouer? Les observations de votre professeur? Les options sont nombreuses, et il en va de même pour les indicateurs de l'ODD 4.

Comment les indicateurs de l'ODD 4 sont-ils élaborés? À l'issue de plusieurs cycles de consultations mondiales et de réunions avec les États membres, les organismes internationaux et régionaux, le milieu universitaire, le monde des affaires, les organisations non gouvernementales (ONG) et la société civile, une liste de 11 indicateurs mondiaux pour le suivi de l'ODD 4 a été officiellement adoptée par l'Assemblée générale des Nations Unies en 2017. Une fois un consensus atteint sur la liste des indicateurs mondiaux de l'éducation, un outil de classification par niveau a été élaboré pour identifier l'état d'élaboration méthodologique de chaque indicateur et la disponibilité des données à l'échelle mondiale. Les méthodologies et les normes des indicateurs de Niveau 1 et de Niveau 2 sont établies à l'échelle internationale, mais les indicateurs de Niveau 2 ne sont pas disponibles dans un nombre suffisant de pays. Les indicateurs de Niveau 3 nécessitent l'élaboration de méthodologies et de normes, et ce travail a été classé prioritaire par le Groupe inter-agences et d'experts sur les indicateurs des ODD (GIAE-ODD), un groupe de 28 experts nationaux en statistique, représentatifs au niveau international, établi par la Commission de statistique des Nations Unies en 2015 pour élaborer et mettre en œuvre un cadre mondial d'indicateurs pour le suivi des ODD. Tous les indicateurs sont jugés d'une importance égale pour le suivi de l'ODD 4, quel que soit leur niveau de classification.

Le Document d'information de l'ISU n $^{\circ} 45$ (2017c) décrit les procédures actuelles entreprises par l'Institut de statistique de l'UNESCO (ISU) pour élaborer les indicateurs de l'éducation à partir des données administratives et des enquêtes. 
Les mêmes indicateurs seront-ils utilisés par tous les pays et toutes les régions? Le suivi de l'ODD 4 est fondé sur des principes universels et met l'accent sur un cadre participatif dans lequel toutes les parties prenantes (société civile, monde des affaires, parlement, milieu universitaire et gouvernement) peuvent reconnaître leur responsabilité commune pour réaliser les ODD. La Figure 1 montre le cadre à niveaux et à objectifs multiples, qui est composé de quatre niveaux de suivi : mondial, thématique, régional et national.

Le suivi mondial repose sur un groupe limité et soigneusement sélectionné d'indicateurs principaux pour donner un aperçu des progrès accomplis vers la réalisation de chaque cible. L'harmonisation du suivi des ODD et l'établissement des rapports à des fins de comparabilité internationale sont aussi d'une importance capitale. La capacité à analyser et à comparer les données nationales entre les pays et les années fournit des renseignements pour mesurer la performance, conduire les réformes politiques et affecter équitablement les ressources pour améliorer l'apprentissage entre tous les groupes de population. Le GIAE-ODD, composé des États membres et incluant des organismes régionaux et internationaux en qualité d'observateurs, est responsable de l'élaboration du cadre mondial d'indicateurs, ainsi que de son perfectionnement et de ses révisions occasionnelles. Le GIAE-ODD a identifié I'ISU comme institution dépositaire de 9 des 11 indicateurs mondiaux de l'ODD 4 et comme organisme partenaire des deux autres indicateurs mondiaux.

Le suivi thématique ajoute un niveau de suivi aux indicateurs comparables au plan transnational dans un secteur spécifique (par ex. éducation, environnement, énergie, santé) ou des thèmes transversaux (par ex. le genre). Les indicateurs thématiques servent de cadre pour surveiller les progrès sur une base comparable au plan transnational, en offrant un aperçu plus approfondi des priorités sectorielles que celui qui est disponible dans le cadre mondial de suivi. Ce niveau donne la possibilité d'identifier les défis et les goulets d'étranglement spécifiques à un secteur et de mobiliser les mesures nécessaires pour s'y attaquer. Le suivi thématique de l'ODD 4 suit les lignes directrices établies par le Cadre d'action Éducation 2030 qui a été adopté par les 184 États membres de l'UNESCO en 2015. Les indicateurs thématiques de l'ODD 4 sont élaborés par le Groupe de coopération technique sur les indicateurs de l'ODD 4-Éducation 2030 (GCT), qui est composé de représentants des États membres, des organismes internationaux et d'organisations de la société civile. L'ISU héberge le Secrétariat et copréside le GCT avec le soutien de la Division Éducation 2030 de I'UNESCO.

Pour le suivi régional, un ensemble d'indicateurs sera élaboré pour examiner les priorités et les questions d'intérêt commun que partagent les pays d'une région particulière, comme indiqué dans les documents de planification ou les cadres régionaux. Différentes régions ou sous-régions sont parvenues à des accords sur certains objectifs et cibles avant même l'approbation des ODD. Une étape cruciale pour promouvoir l'efficience et éviter la duplication des efforts consiste à cartographier les stratégies mondiales et régionales. L'ISU et le Rapport mondial de suivi sur l'éducation (GEMR) ont produit un aperçu mondial des mécanismes régionaux de suivi, d'établissement des rapports et de comparaison de l'éducation :

Asie de l'Est et Pacifique: Association des nations d'Asie du Sud-Est (ANASE) : Work Plan on Education 2016-2020 (Plan de travail pour l'éducation 2016-2020) ; Forum des îles du Pacifique (FIP) : Pacific Education Development Framework (Cadre de développement de l'éducation pour le Pacifique) ; Southeast Asia Ministers of Education Organization (SEAMEO) (Organisation des ministres de l'Éducation d'Asie du Sud-Est) : Education Agenda 2035 (Agenda Éducation 2035) ; Communauté du Pacifique (CPS) : Education Quality Assessment Programme (Programme pour l'évaluation et la qualité de l'enseignement). 
Europe et Amérique du Nord : Union européenne (UE) : Éducation et formation 2020 (EF 2020).

Amérique latine et Caraïbes : Communauté des Carailbes (CARICOM) : Human Resource Development 2030 Strategy (Stratégie de développement des ressources humaines 2030) ; Conseil éducationnel et culturel d'Amérique centrale (CECC) : Central American Education Policy (PEC) (Politique d'éducation de l'Amérique centrale) ; Organisation des États américains (OEA) : Inter-American Education Agenda (Programme interaméricain de l'éducation) ; Organisation des États des Carailbes orientales (OECO) : OECS Education Sector Strategy (Stratégie sectorielle de l'éducation de l'OECO) ; Organisation des États ibéroaméricains (OEI) : Metas 2021 (Objectifs de l'éducation 2021).

Afrique du Nord et Asie de l'Ouest: Union africaine (UA) : Stratégie continentale de l'éducation pour l'Afrique 2016-2025 ; Organisation de la Ligue arabe pour l'éducation, la culture et les sciences (ALECSO).

Asie du Sud et de l'Ouest: Association sud-asiatique de coopération régionale (ASACR) : SAARC Development Goals: Taking SDGs Forward (Objectifs de développement de l'ASACR : faire avancer les ODD).

Afrique subsaharienne : Union africaine (UA) : Stratégie continentale de l'éducation pour l'Afrique 20162025 ; Communauté de développement de l'Afrique australe (CDAA) : Process of update of Protocol on Education and Training/Regional Implementation Plan 2007-2015 (Processus de mise à jour du Protocole sur l'éducation et la formation/Plan régional de mise en œuvre 2007-2015).

Organisations interrégionales: Commonwealth : Commonwealth Education Policy Framework (Cadre stratégique de l'éducation du Commonwealth) ; Organisation islamique pour l'éducation, la science et la culture (ISESCO) : Strategy for the Development of Education in the Islamic World (Stratégie de développement de l'éducation dans le monde islamique) ; Organisation internationale de la Francophonie (OIF) ; Organisation de coopération et de développement économiques (OCDE).

Le suivi national de l'ODD 4 est lié aux besoins des gouvernements nationaux et infranationaux en matière d'élaboration des plans sectoriels d'éducation et d'éclairage des politiques. Les données qui ont un degré élevé de granularité et qui s'adaptent aux particularités du contexte national (comme les unités géographiques infranationales, les groupes défavorisés spécifiques ou par richesse) offrent une plus grande capacité à éclairer les politiques en examinant les disparités pertinentes dans les résultats d'apprentissage. Le suivi de l'ODD 4 à ce niveau bénéficie de la participation active d'un groupe divers de parties prenantes représentant leurs groupes respectifs et les préoccupations liées à l'éducation. L'ISU travaille aussi en étroite collaboration avec les gouvernements nationaux et les instituts de la statistique pour soutenir les stratégies nationales de collecte des données et permettre l'établissement des rapports internationaux sur toutes les cibles de l'ODD 4 au sein du Cadre d'action Éducation 2030. 
Figure 1. Les quatre niveaux de suivi des cibles de l'éducation

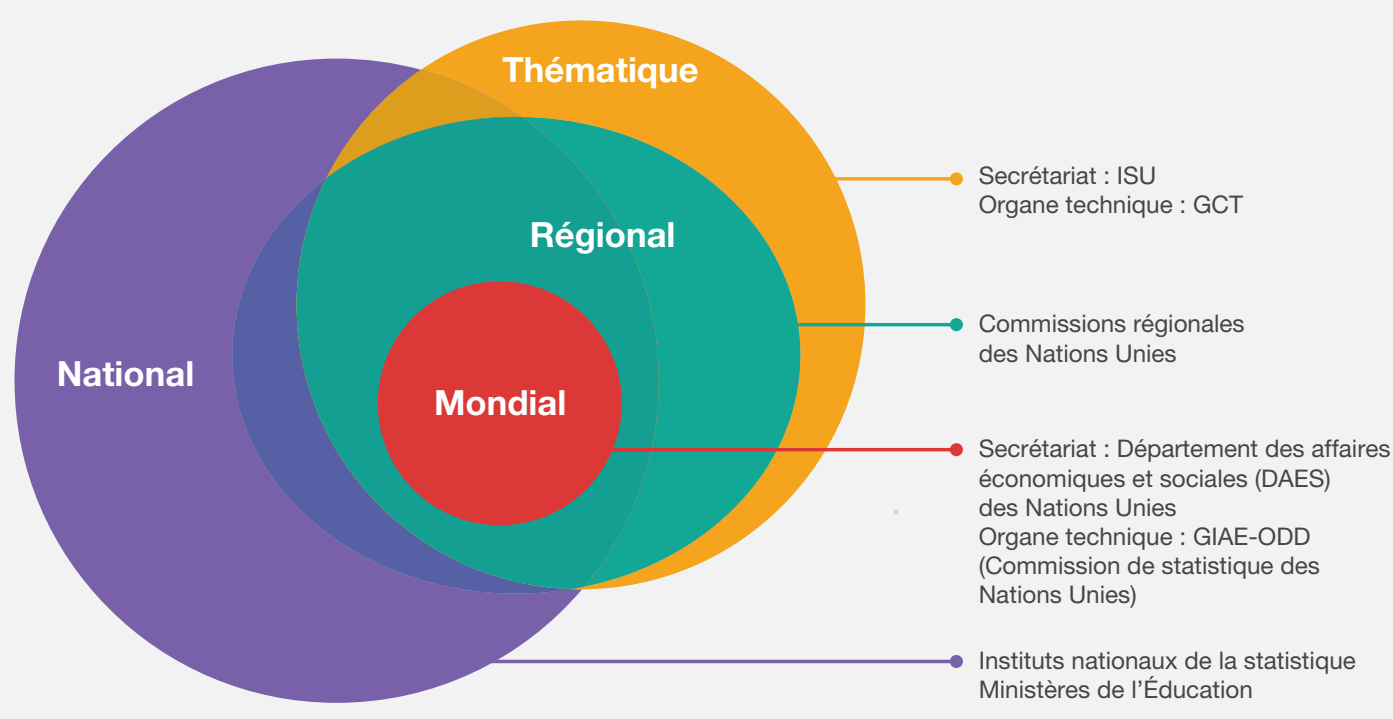

Source : Institut de statistique de l'UNESCO, 2016

Les indicateurs mondiaux et thématiques de l'éducation ont été conçus pour faciliter le suivi transnational des progrès accomplis vers la réalisation des cibles. Les pays seront encouragés à établir des rapports sur les indicateurs mondiaux et thématiques. Chaque pays déterminera s'il est en mesure de collecter toutes les données nécessaires pour chacun des indicateurs recommandés et d'établir les rapports comme demandé. Les pays peuvent choisir à partir de la liste des indicateurs thématiques les plus pertinents pour leurs besoins politiques. Les organisations internationales continueront à collecter des données auprès des pays à des fins de comparaisons transnationales et rendre compte des tendances. 



\section{Quels sont les outils existants pour mesurer les progrès accomplis vers la réalisation de l'ODD 4 ?}

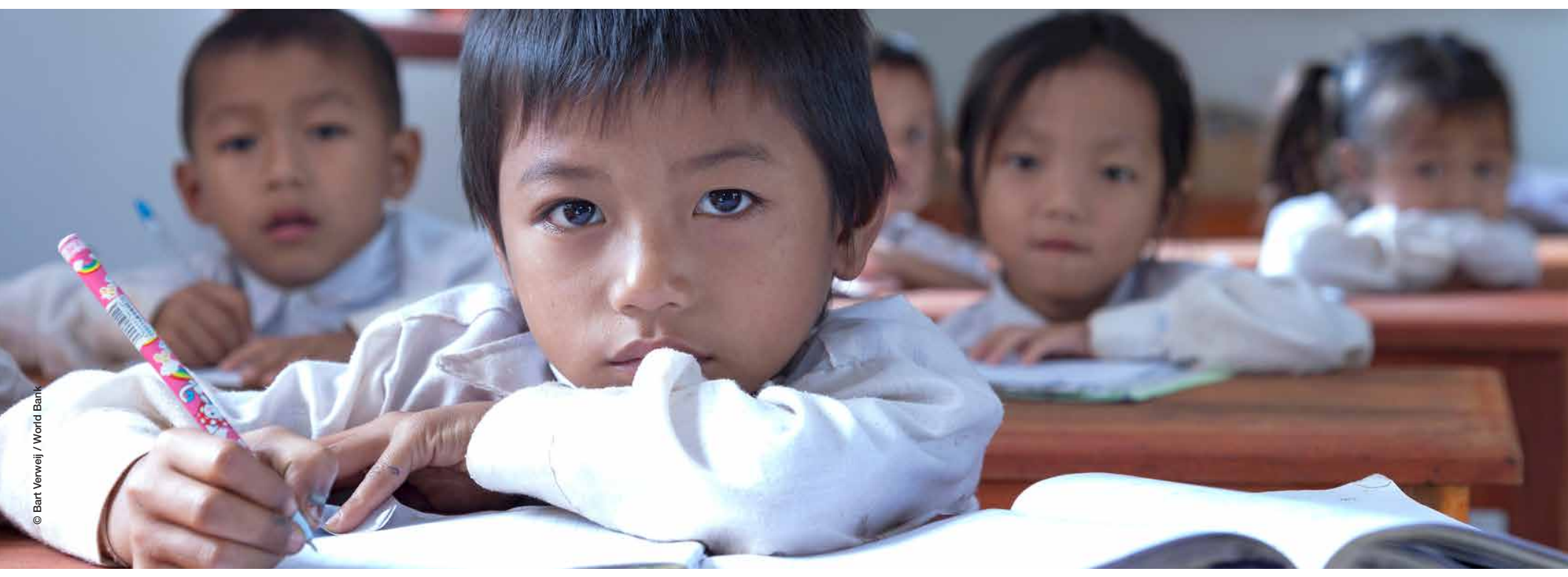

II existe plusieurs organisations régionales et internationales qui génèrent des informations sur les méthodes pour mesurer les progrès accomplis par les pays vers la réalisation des cibles de l'ODD 4. La responsabilité de la coordination des données de l'ODD et l'élaboration d'indicateurs au niveau international incombe aux institutions dépositaires de l'ODD.

Les institutions dépositaires sont les organismes des Nations Unies (et dans certains cas d'autres organisations internationales) responsables de compiler et de vérifier les données et les métadonnées des pays, et de communiquer à la Division de la statistique des Nations Unies (DSNU) les données ainsi que les agrégats régionaux et mondiaux. Les institutions assurent la comparabilité des données au plan international et élaborent les normes et les méthodologies internationales pour aider les pays à effectuer le suivi. Les données au niveau national peuvent être publiées dans leurs bases de données et utilisées pour établir des rapports thématiques.

\section{Le réseau institutionnel}

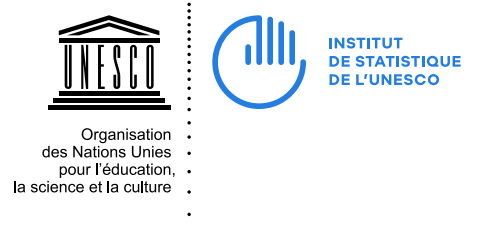

En tant que source officielle de données comparables au plan international, I'ISU a reçu le mandat de suivre les progrès accomplis vers la réalisation de l'ODD 4 sur l'éducation et des cibles clés associées à la science et à la culture. Comme défini dans la Cadre d'action Éducation 2030, I'ISU travaille avec les organisations partenaires et les experts à l'élaboration de nouveaux 
indicateurs, d'approches statistiques et d'outils de suivi pour évaluer les progrès accomplis vers la réalisation de I'ODD 4 (UNESCO, 2016), en coordination avec le Comité directeur ODD-Éducation 2030.

Ce mandat témoigne de la confiance que la communauté internationale accorde aux données de l'ISU et à son expérience avérée en matière de travail méthodologique et de définition de normes avec les instituts nationaux de la statistique, les ministères concernés et les partenaires techniques de chaque région.

Citons parmi les principales initiatives de l'ISU :

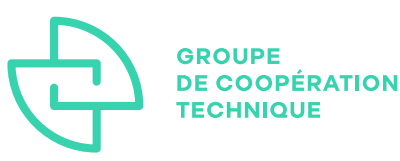

Le Groupe de coopération technique sur les indicateurs de I'ODD 4-Éducation 2030 (GCT) sert de plateforme pour discuter et élaborer les indicateurs utilisés pour suivre les cibles de l'ODD 4 de manière ouverte, inclusive et transparente. Le GCT est composé de 38 experts en statistique, représentatifs au plan régional, issus des États membres, des organisations internationales, de la société civile et du co-président du Comité directeur ODD-Éducation 2030.

ALLIANCE

MONDIALE POUR

LE SUIVI DE

L'APPRENTISSAGE
L'Alliance mondiale pour le suivi de l'apprentissage (GAML) est conçue pour améliorer les résultats d'apprentissage en soutenantles stratégies nationales d'évaluations de l'apprentissage et en élaborant des indicateurs et des outils méthodologiques comparables au plan international pour mesurer les progrès accomplis vers la réalisation

des cibles clés de l'ODD 4. Grâce à une approche hautement collaborative, la GAML réunit un large éventail de parties prenantes, incluant les experts et les décideurs impliqués dans les initiatives nationales et transnationales d'évaluations de l'apprentissage, ainsi que les donateurs et les organisations de la société civile.

Le Groupe inter-agences sur les indicateurs de l'inégalité dans l'éducation (GIA-IIE) est établi pour promouvoir et coordonner l'utilisation des données provenant des enquêtes auprès des ménages pour le suivi de l'éducation au niveau national, régional et mondial. Le GIA-IIE est dirigé par I'ISU, I'UNICEF et la Banque mondiale et comprend également des membres provenant d'autres organisations impliquées dans la production et l'utilisation des données provenant des enquêtes auprès des ménages : le GEMR, le Partenariat mondial pour l'éducation (PME), I'ICF, I'OCDE, RTI International et l'Agence des États-Unis pour le développement international (USAID).

Le système des Nations Unies a développé un vaste réseau de soutien pour le suivi des ODD au niveau mondial, régional et national. Le Tableau 1 décrit en détail les rôles spécifiques des organisations à chaque niveau.

\section{Sources de données}

Les pays sont le point de départ de tout suivi national et international. Les acteurs impliqués dans la collecte et la diffusion des données incluent les instituts nationaux de la statistique, les ministères responsables et les autres institutions nationales pertinentes.

Les pays déterminent le niveau de détail des données et des métadonnées qu'ils partagent avec les institutions dépositaires et la quantité qui sera publiée. Plus les données sont ventilées, plus elles sont utiles à un large éventail de publics. Les indicateurs mondiaux des ODD ne représentent qu'un sous-ensemble du jeu complet 
Tableau 1. Rôle de soutien du système des Nations Unies, des organisations internationales et des principaux groupes

\section{Mondial}

Secrétaire général des Nations Unies

- Rapport mondial sur les ODD

- Rapport des travaux de l'organisation

Conseil des chefs de Secrétariat des Nations Unies

- Mettre en conformité les activités avec les ODD et les défis émergents

- Cohérence politique au sein du système des Nations Unies

Groupe inter-agences et d'experts sur les ODD et Département des affaires économiques et sociales des Nations Unies

- Élaboration des indicateurs

- Rapport annuel sur l'état d'avancement

- SDMX, données géospatiales

Autres entités du Secrétariat des Nations Unies, institutions spécialisées des Nations Unies et organisations du système des Nations Unies

- Examens/analyses thématiques

Autres organismes multilatéraux et groupes principaux

- Examens/analyses thématiques

Groupe de développement des Nations Unies

- Rapports/examens sur l'état d'avancement des pays

- Rapports/examens nationaux thématiques

\section{Régional}

Commissions régionales

- Forums régionaux sur le développement durable

- Rapports régionaux sur les ODD

Mécanisme régional de coordination

- Coordination des programmes

- Cohérence politique

\section{Organisations régionales}

intergouvernementales

$\checkmark$ Nouveau partenariat pour le développement de l'Afrique

$\checkmark$ Association des nations d'Asie du Sud-Est

$\checkmark$ Association d'Asie du Sud pour la coopération régionale, etc.

- Revues par les pairs

- Partage des leçons apprises

Banques régionales de développement $\checkmark$ Banque asiatique de développement $\checkmark$ Banque africaine de développement $\checkmark$ Nouvelle banque de développement

- Examens/analyses thématiques

- Harmonisation du prêt

\section{Groupe de développement des} Nations Unies

- Examens programmatiques régionaux/ nationaux

- Examens régionaux et développement des capacités

\section{National}

Équipe de pays des Nations Unies

- Soutient les consultations et les examens nationaux/infranationaux multipartites

- Joue un rôle d'honnête intermédiaire pour réunir autour d'une table les différentes directions ministérielles et les groupes principaux pour accroître la coordination et la cohérence politique

- Soutient l'intégration des ODD et la personnalisation nationale

- Soutient le renforcement de la base de données factuelles (par ex. évaluations des goulets d'étranglement)

- Soutient la préparation de l'établissement des rapports nationaux sur les ODD

- Soutient l'organisation et la coordination des activités de renforcement des capacités

- Soutient la collecte, l'analyse et la synthèse des données et des éléments d'informations relatifs aux ODD

\section{Groupes principaux/société civile}

- Examens thématiques/sectoriels nationaux/ infranationaux

- Examens pour accroître l'appropriation nationale et la responsabilisation

Groupe de développement des Nations Unies

- Fournit des orientations à l'équipe de pays des Nations Unies en soutien aux ODD

Source : Groupe de développement des Nations Unies (GDNU), 2017. 
des indicateurs suivis dans un pays. Les pays peuvent partager des jeux supplémentaires de données avec les institutions dépositaires et le Forum politique de haut niveau pour le développement durable (FPHN) des Nations Unies, par exemple par le biais d'examens nationaux volontaires.

Données administratives. Chaque année, I'ISU mène des enquêtes sur les programmes d'enseignement formel et le niveau d'éducation atteint, et tous les deux ans, une enquête sur l'alphabétisme. Ces enquêtes consistent en une série de questionnaires sur les programmes éducatifs, les élèves, les ressources financières et humaines, l'alphabétisme et le niveau d'éducation atteint, et ces données sont communiquées conformément aux normes internationales pour assurer la comparabilité entre les pays. Les principales sources de données sont les documents administratifs des enquêtes menées en milieu scolaire ou les données agrégées des enquêtes auprès de la population active ou auprès des ménages. Les questionnaires sont envoyés aux instituts nationaux de la statistique et aux ministères de l'Éducation de chaque pays. Les résultats de ces questionnaires sont la source de nombreux indicateurs de l'ODD 4.

Les données sur le financement et les dépenses de l'éducation sont essentielles pour aborder efficacement les questions essentielles de la politique éducative. Les données sur les dépenses publiques et internationales pour l'éducation sont dérivées des documents administratifs compilés généralement par le ministère des Finances, le ministère de l'Éducation ou les instituts nationaux de la statistique. En 2016, l'Institut international de planification de l'éducation (IIPE) de I'UNESCO et l'ISU ont élaboré conjointement une méthodologie sur les Comptes nationaux de l'éducation (CNE), un cadre complet, systématique et comparable de collecte, de traitement et d'analyse des données sur les dépenses de l'éducation (IIPE, ISU et Pôle de Dakar, 2016). Les données sur les dépenses privées des ménages sont dérivées des enquêtes sur le budget des ménages, habituellement administrées par les instituts nationaux de la statistique.

Enquêtes auprès des ménages. L'ISU compile et diffuse des données provenant des enquêtes auprès des ménages pour produire des indicateurs afin d'examiner des caractéristiques spécifiques des populations, qui ne sont généralement disponibles qu'à travers ces sources. Les ensembles de données des enquêtes auprès des ménages proviennent de programmes internationaux comme l'Enquête démographique et de santé (EDS) et I'Enquête par grappes à indicateurs multiples (MICS), ainsi que du répertoire d'enquêtes d'organisations comme la Commission économique pour l'Amérique latine et les Caraïbes (CEPALC) des Nations Unies, des Séries de microdonnées intégrées à usage public (IPUMS) et de la Banque mondiale. Ces données sont utilisées pour calculer différents indicateurs de l'éducation, comme les taux de fréquentation scolaire et d'achèvement.

Évaluations de l'apprentissage. L'ISU a identifié neuf évaluations transnationales de l'apprentissage qui remplissent les critères pour mesurer l'Indicateur 4.1.1 de l'ODD 4 (pourcentage d'enfants et de jeunes qui maîtrisent au moins les normes d'aptitudes minimales en lecture et en mathématiques) : LaNA (Évaluation des aptitudes à la lecture, à l'écriture et au calcul), PASEC (Programme d'analyse des systèmes éducatifs de la CONFEMEN), PILNA (Évaluation des aptitudes à la lecture, à l'écriture et au calcul dans les îles du Pacifique), PIRLS (Programme international de recherche en lecture scolaire), PISA (Programme international pour le suivi des acquis des élèves), SACMEQ (Consortium de l'Afrique australe et orientale pour le pilotage de la qualité de l'éducation), SEA-PLM (Métriques de l'apprentissage à l'école primaire en Asie du Sud-Est), TERCE (Troisième étude régionale comparative et explicative) et TIMSS (Tendances de l'enquête internationale sur les mathématiques et les sciences). À l'aide de ces sources de données, l'ISU a calculé les indicateurs clés liés aux résultats d'apprentissage de l'ODD 4. 
L'ISU (2018) a rédigé une proposition de plateforme centrée sur l'éducation appelée edu2030/countrySTAT sous la forme d'un réseau mondial de partage des données qui place les pays au centre de l'initiative et aborde leurs besoins de développement des données pour l'ODD 4. Facilement accessible en ligne, il permettrait aux chercheurs, aux décideurs politiques, aux organismes de développement et au secteur privé de concevoir et mettre en œuvre de meilleures politiques et de réduire les coûts de transaction et les lacunes en matière d'information.

\section{Principales plateformes régionales}

De nombreuses régions disposent de structures internationales pour suivre le développement de l'éducation dans les pays. Elles constituent des sources importantes de données comparables au plan international avec un degré élevé de pertinence régionale. Citons parmi ces initiatives régionales :

\section{Afrique}

Le PASEC a été administré dans 13 pays francophones d'Afrique de l'Ouest. Le PASEC est conçu pour évaluer les aptitudes des élèves en mathématiques et en lecture en français. Commencé en 1993, le programme est géré par la Conférence des ministres de l'Éducation des États et gouvernements de la Francophonie (CONFEMEN). L'évaluation est administrée pour différentes années dans différents pays. Le PASEC est généralement administré aux élèves de $2 e$ et de $5 e$ année d'études au début et à la fin de la même année scolaire pour mesurer les progrès des élèves au cours de l'année. Les résultats de l'évaluation sont destinés à être utilisés principalement comme un outil de diagnostic.

Le SACMEQ est un consortium des ministères de l'Éducation situés dans la sous-région d'Afrique australe. Le SACMEQ vise à évaluer et à suivre la qualité de l'éducation et les acquis de l'apprentissage dans les pays membres. Les grands domaines de l'évaluation sont les caractéristiques des élèves et leurs milieux d'apprentissage, les caractéristiques des enseignants, les caractéristiques des chefs d'établissement et leurs points de vue sur l'infrastructure scolaire et la gestion. Le SACMEQ a mené des évaluations en 1995, en 2000 et en 2006. La mise en œuvre du quatrième cycle d'évaluation devait commencer en 2012.

Uwezo, qui signifie "capacité» en kiswahili, est une initiative quinquennale qui vise à améliorer les compétences en lecture, en écriture et en calcul des enfants âgés de 6 à 16 ans au Kenya, en Tanzanie et en Ouganda. Depuis 2009, l'Uwezo a mis en œuvre des enquêtes à grande échelle auprès de ménages représentatifs au plan national afin d'évaluer les compétences de base réelles en lecture, en écriture et en calcul des élèves d'âge scolaire. Chaque année, l'Uwezo produit trois rapports nationaux et un rapport régional qui présente les principales conclusions.

\section{États Arabes}

Contrairement aux autres régions de l'UNESCO, les États arabes n'utilisent pas une approche harmonisée au plan régional pour mesurer les acquis des élèves. De plus, de nombreux pays dépendent uniquement des examens de fin de cycle. Néanmoins, davantage de pays participent aux évaluations à grande échelle, comme TIMSS et PIRLS dirigées par l'IEA, en plus de PISA dirigée par l'OCDE. 


\section{Asie et le Pacifique}

SEA-PLM est une évaluation régionale qui tente d'établir une approche commune pour évaluer les résultats d'apprentissage des élèves de $5 e$ année d'études en lecture, écriture, mathématiques et citoyenneté mondiale. Le Secrétariat de l'Organisation des ministres de l'Éducation d'Asie du Sud-Est (SEAMEO) et le Bureau régional de l'Asie orientale et Pacifique de l'UNICEF (EAPRO) servent conjointement de secrétariat à SEA-PLM.

Le mandat de l'initiative PILNA a été établi par les ministres de l'Éducation du Forum des îles du Pacifique durant la réunion du Forum des ministres de l'Éducation en Papouasie Nouvelle-Guinée en 2010. PILNA a été administrée dans 14 îles du Pacifique en 2012 pour définir la référence de base régionale et le classement des pays en lecture, écriture et calcul des élèves qui ont achevé quatre et six années d'enseignement primaire.

Le Réseau sur le suivi de la qualité de l'éducation en Asie-Pacifique (NEQMAP), établi en mars 2013 à Bangkok, est une plateforme pour échanger les connaissances, les expériences et l'expertise sur le suivi de la qualité de l'éducation dans les pays et les juridictions de la région Asie-Pacifique. Le réseau est centré sur les évaluations de l'apprentissage des élèves en tant qu'outils clés pour le suivi de la qualité de l'éducation, tout en reconnaissant l'importance du rôle du programme d'étude et de la pédagogie pour un apprentissage réussi. Le Bureau régional pour l'éducation en Asie et dans le Pacifique de l'UNESCO (UNESCO Bangkok) sert de secrétariat à NEQMAP.

\section{Europe et Amérique du Nord}

Eurostat est l'institut de statistique de l'UE situé au Luxembourg. II a pour mission de fournir des statistiques de haute qualité aux pays européens. Les statistiques de l'éducation et de la formation d'Eurostat fournissent des informations sur la participation des individus aux activités d'éducation et de formation, le financement de l'éducation, le personnel enseignant et les résultats scolaires. Bien que la plupart des pays d'Europe et d'Amérique du Nord n'aient pas d'évaluations régionales spécifiques de l'apprentissage, ils participent aux évaluations internationales à grande échelle organisées par l'IEA et l'OCDE.

\section{Amérique latine et Caraïbes}

Le Laboratoire latino-américain d'évaluation de la qualité de l'éducation (LLECE), dont le siège se trouve au Bureau régional pour l'éducation en Amérique latine et dans les Carailbes de l'UNESCO, est un réseau d'unités nationales mesurant l'évaluation de l'éducation dans la région. LLECE mène des études comparatives et exploratoires régionales d'évaluation de l'éducation depuis 1997.

Le Catalogue sur les évaluations de l'apprentissage (CEA) de l'ISU fournit des informations descriptives, normalisées et comparables sur les examens publics, les évaluations nationales et internationales qui sont administrés dans les programmes du primaire et du premier cycle du secondaire dans les pays à travers le monde. Le Catalogue sert de ressource aux pays qui souhaitent élaborer une évaluation nationale, améliorer leur système général d'évaluation ou faire partie d'une initiative régionale ou internationale. La seconde version du Catalogue a été élargie pour inclure non seulement les évaluations de l'apprentissage dans l'enseignement primaire et secondaire, mais aussi les évaluations sur les compétences des jeunes et des adultes et le développement de la petite enfance. 


\section{Quel est le rôle de l'ISU dans le suivi de l'ODD 4 ?}

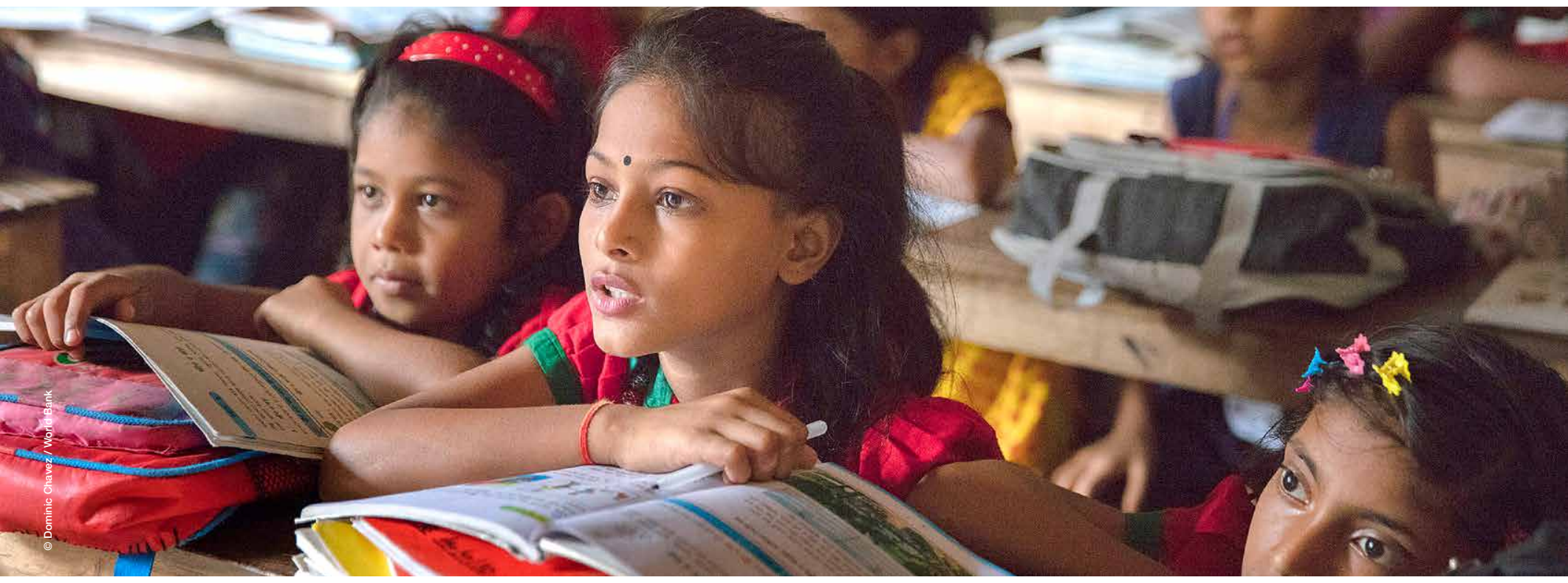

L'ISU est l'agence statistique officielle de l'UNESCO. À ce titre, la communauté internationale du développement a confié à l'ISU le mandat d'élaborer les méthodologies, les normes et les indicateurs nécessaires pour réaliser I'ODD 4-Éducation 2030 et les cibles clés en matière de science et de culture, en étroite consultation avec les partenaires. Les initiatives de l'ISU soutiennent les activités dans plusieurs domaines de suivi de l'ODD 4 (voir la Figure 2).

\section{Rapports intermédiaires}

Le suivi des 43 indicateurs mondiaux et thématiques de l'éducation suit une stratégie similaire d'établissement des rapports pour toutes les cibles de l'ODD. Cependant, certaines données pour calculer les indicateurs ne sont pas disponibles actuellement. Bien que I'ISU travaille au renforcement des capacités et à l'élaboration des indicateurs, des sources de données alternatives sont utilisées pendant la période intermédiaire pour maximiser la disponibilité des données. L'objectif est de rendre compte des progrès accomplis avant la finalisation de l'élaboration méthodologique des principales sources de données. Les stratégies d'établissement des rapports intermédiaires seront appliquées jusqu'à ce que des stratégies d'établissement des rapports durables et à long terme soient mises en place pour chacun des indicateurs.

\section{Collaboration}

En tant que source officielle de données comparables au plan international, l'ISU porte la responsabilité principale de la réussite du suivi de l'ODD 4. Cette responsabilité est partagée avec les organisations partenaires. Bien que I'ISU ait reçu le mandat de produire les données nécessaires pour le suivi des progrès accomplis et de diriger les politiques et les ressources vers ceux qui en ont le plus besoin, aucune organisation ne peut produire seule 
la totalité des données de tous les indicateurs. Pour certains indicateurs mondiaux, par exemple, différentes organisations ont été désignées comme institution dépositaire (voir le Tableau 2).

\section{Figure 2. Rôle de I'ISU dans le suivi de I'ODD 4}

\section{RENFORCER LES CAPACITÉS}

La capacité de la plupart des pays en matière de statistique est mise à l'épreuve par l'étendue, la profondeur et l'ambition de l'objectif mondial d'éducation. L'accent mis sur l'équité et la qualité de l'éducation dans l'Agenda de développement durable 2030 et la nature plus complète des ODD par rapport aux OMD nécessitent plus de données qu'auparavant et un éventail plus large de sources : données administratives, données financières, données issues de recensements, d'enquêtes auprès des ménages, et d'évaluations nationales, régionales et internationales de l'apprentissage. Fort de sa longue expérience de travail avec les pays, l'ISU a élaboré un ensemble d'outils et de stratégies pour aider les instituts nationaux de la statistique et les ministères concernés à surmonter les défis de la mesure. Le Rapport 2017 sur les données de I'ODD 4 de l'ISU décrit ces stratégies en se concentrant sur la qualité des données en tant que fondement d'un cadre efficace de suivi de l'ODD 4 (ISU, 2017b).

\section{DÉFINIR LA NORME}

Au niveau international, il est essentiel de s'assurer que les « statistiques des pommes " ne soient pas mélangées aux "statistiques des oranges ». À cet effet, l'ISU élabore des normes et les met en œuvre pour assurer la comparabilité internationale des données. En même temps, I'Institut travaille à améliorer la qualité des données produites pour suivre l'éducation à travers le monde. La Classification internationale type de l'éducation (CITE) et les travaux de la GAML pour définir des métriques de l'apprentissage ou des échelles comparables pour les cibles pertinentes de l'ODD 4 en sont deux exemples.

\section{ÉLABORER LES INDICATEURS}

L'élaboration d'indicateurs est le cœur des travaux de l'ISU et l'un des moteurs principaux de l'introduction de nouvelles collectes de données ou de la modification de celles qui existent. Une fois que le besoin d'un indicateur est identifié et que son élaboration est validée, les prochaines étapes consistent à élaborer la méthodologie pour calculer l'indicateur, identifier les sources de données, administrer une enquête auprès des États membres ou identifier des sources alternatives de données, et enfin, analyser les indicateurs (ISU, 2017c). Pour le suivi de l'ODD 4, le GCT et la GAML sont les principales tribunes pour le travail d'élaboration des indicateurs basé sur la participation active des États membres, des organisations partenaires et des experts de premier plan.

\section{COLLECTER LES DONNÉES}

L'ISU compile les données et les métadonnées relatives à l'éducation provenant de différentes sources au niveau national, régional et international. Bien que la plupart des données soient collectées par le biais des questionnaires annuels de l'ISU envoyés aux États membres, l'Institut produit également des indicateurs basés sur des enquêtes auprès des ménages et des évaluations internationales de l'apprentissage.

\section{DIFFUSER ET ANALYSER}

La plupart des activités ne peuvent avoir un impact efficace sur l'éducation que si les informations produites sont utilisées pour améliorer les politiques et les pratiques éducatives. L'ISU s'est engagé à diffuser ses données largement pour permettre une prise de décision politique fondée sur des données factuelles. Au niveau mondial, I'ISU travaille avec d'autres institutions des Nations Unies pour offrir une perspective globale et cohérente des ODD. Une étroite collaboration avec le GEMR est en place pour permettre l'analyse complète des données factuelles produites par le cadre de suivi. 
Tableau 2. Indicateurs mondiaux de l'ODD 4 et institutions dépositaires

\begin{tabular}{|c|c|c|}
\hline & Indicateur & $\begin{array}{l}\text { Institution } \\
\text { dépositaire }\end{array}$ \\
\hline 4.1.1 & $\begin{array}{l}\text { Pourcentage d'enfants et de jeunes : (a) en } 2 e \text { ou } 3 e \text { année d'études ; (b) en fin de cycle } \\
\text { primaire; et (c) en fin de premier cycle du secondaire qui maîtrisent au moins les normes } \\
\text { d'aptitudes minimales en (i) lecture et (ii) mathématiques, par sexe }\end{array}$ & UNESCO-ISU \\
\hline 4.2 .1 & $\begin{array}{l}\text { Pourcentage d'enfants de moins de cinq ans dont le développement est en bonne voie en } \\
\text { matière de santé, d'apprentissage et de bien-être psychosocial, par sexe }\end{array}$ & UNICEF \\
\hline 4.2 .2 & $\begin{array}{l}\text { Taux de participation à des activités organisées d'apprentissage (un an avant l'âge officiel } \\
\text { de scolarisation dans le primaire), par sexe }\end{array}$ & UNESCO-ISU \\
\hline 4.3.1 & $\begin{array}{l}\text { Pourcentage de jeunes et d'adultes ayant participé à un programme d'éducation et de } \\
\text { formation formelle ou non formelle au cours des } 12 \text { derniers mois, par sexe }\end{array}$ & UNESCO-ISU \\
\hline 4.4.1 & $\begin{array}{l}\text { Pourcentage de jeunes et d'adultes ayant des compétences en matière de technologies de } \\
\text { l'information et de la communication (TIC), par type de compétence }\end{array}$ & $\begin{array}{l}\text { UNESCO-ISU, } \\
\text { UIT }\end{array}$ \\
\hline 4.5.1 & $\begin{array}{l}\text { Indices de parité (femmes/hommes, urbain/rural, quintile inférieur/supérieur de richesse et } \\
\text { autres paramètres tels que le handicap, le statut d'autochtone et les situations de conflit, à } \\
\text { mesure que les données deviennent disponibles) pour tous les indicateurs de l'éducation } \\
\text { de cette liste pouvant être ventilés }\end{array}$ & UNESCO-ISU \\
\hline 4.6 .1 & $\begin{array}{l}\text { Pourcentage de la population d'une tranche d'âge donnée atteignant au moins un certain } \\
\text { niveau de maîtrise de compétences fonctionnelles en matière (a) de lecture et d'écriture et } \\
\text { (b) de calcul, par sexe }\end{array}$ & UNESCO-ISU \\
\hline 4.7.1 & $\begin{array}{l}\text { Mesure dans laquelle (i) l'éducation à la citoyenneté mondiale et (ii) l'éducation en vue } \\
\text { du développement durable, y compris l'égalité entre les sexes et les droits de l'homme, } \\
\text { sont intégrées à tous les niveaux dans : (a) les politiques nationales d'éducation, (b) les } \\
\text { programmes scolaires, (c) la formation des enseignants et (d) l'évaluation des élèves }\end{array}$ & UNESCO-ISU \\
\hline 4.a.1 & $\begin{array}{l}\text { Pourcentage d'écoles disposant : (i) de l'électricité ; (ii) de l'Internet à des fins } \\
\text { pédagogiques ; (iii) d'ordinateurs à des fins pédagogiques ; (iv) d'infrastructures et de } \\
\text { matériels adaptés aux élèves handicapés ; (v) d'un accès élémentaire à l'eau potable ; (vi) } \\
\text { d'installations sanitaires de base séparées pour hommes et femmes ; et (vii) d'un minimum } \\
\text { de lavabos }\end{array}$ & UNESCO-ISU \\
\hline 4.b. 1 & $\begin{array}{l}\text { Volume de l'aide publique au développement consacrée aux bourses d'études, par secteur } \\
\text { et type de formation }\end{array}$ & OCDE \\
\hline 4.c.1 & $\begin{array}{l}\text { Pourcentage d'enseignants dans : (a) le préscolaire ; (b) le cycle primaire ; (c) le premier cycle } \\
\text { du secondaire ; et (d) le second cycle du secondaire qui ont au moins reçu (avant leur entrée } \\
\text { en fonction ou en cours d'activité) les formations minimum organisées pour les enseignants } \\
\text { (notamment dans le domaine pédagogique), requises pour l'enseignement à un niveau } \\
\text { pertinent dans un pays donné }\end{array}$ & UNESCO-ISU \\
\hline
\end{tabular}

De plus, I'ISU collabore avec les États membres et les organisations représentant la société civile pour optimiser les efforts et garantir un cadre de suivi inclusif et participatif. À cet effet, l'ISU a noué des partenariats mondiaux et des alliances qui sont essentielles à la réussite du suivi de l'ODD 4. 


\section{Comment les indicateurs de I'ODD 4 sont-ils élaborés et calculés?}

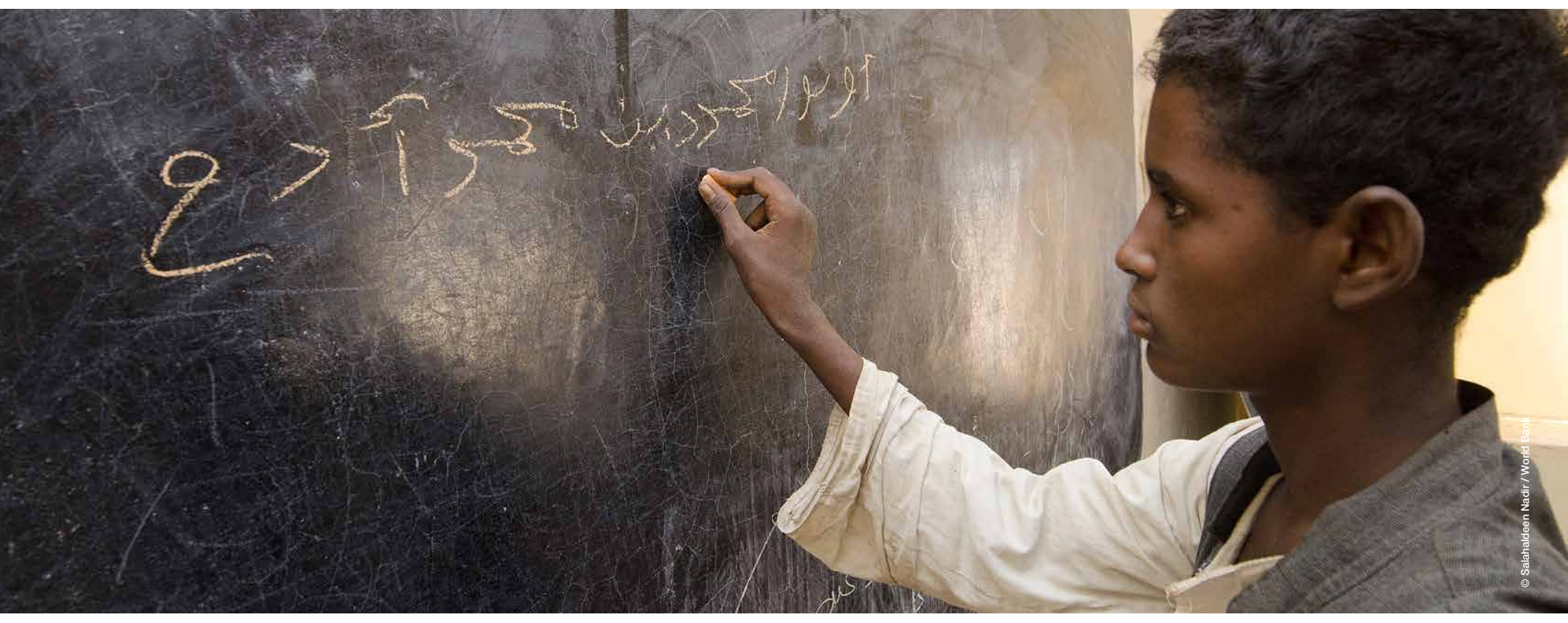

Pour définir les indicateurs mondiaux et thématiques, chaque cible a été analysée avec deux objectifs à l'esprit : identifier les concepts clés qui doivent être mesurés pour suivre les progrès accomplis en vue de sa réalisation, et savoir si les politiques existantes seront suffisantes pour s'assurer que la cible pourra être atteinte ou si d'autres mesures, notamment des mesures correctives, pourraient être nécessaires pour revenir sur la bonne voie. Les concepts clés sous-jacents qui ont guidé l'élaboration des indicateurs étaient les suivants :

\section{Apprentissage, compétences et connaissances}

Cinq des dix cibles de l'éducation sont centrées sur les résultats d'apprentissage des enfants, des jeunes et des adultes. C'est un changement par rapport aux cibles mondiales antérieures, comme les OMD, qui visaient seulement à assurer l'accès, la participation, et l'achèvement dans l'enseignement primaire formel et l'égalité des genres dans les enseignements primaire, secondaire et supérieur. Les cibles de l'Agenda Éducation 2030 soulignent combien la scolarisation et la participation sont les meilleurs moyens pour obtenir de bons résultats et atteindre les résultats d'apprentissage escomptés à tous les âges et à toutes les étapes, comme l'état de préparation à l'école des enfants d'âge préscolaire, les compétences académiques des enfants de l'enseignement primaire et secondaire, les compétences fonctionnelles en lecture, écriture et calcul des jeunes et des adultes, et les compétences utiles pour le travail. 


\section{Équité}

Dans chaque cible, l'objectif primordial sur l'équité vise à s'assurer que personne ne soit laissé pour compte, en particulier les groupes les plus pauvres et les plus vulnérables. Durant les années qui restent jusqu'à 2030, la communauté internationale doit transformer cet engagement décisif en réalité pour tous les enfants, les jeunes et les adultes, quel que soit l'endroit où ils vivent ou leur situation économique. Les indicateurs de l'équité englobent les caractéristiques individuelles, comme le sexe, le lieu de résidence, l'appartenance ethnique, la langue, le handicap et la participation au travail des enfants, ainsi que les caractéristiques des ménages, comme le niveau de scolarité des parents, la richesse ou d'autres mesures du statut socio-économique.

\section{Participation et achèvement}

Les indicateurs sur la participation et l'achèvement ont les taux les plus élevés de disponibilité des données parmi les pays. Ceci s'explique probablement par la disponibilité de ces données dans les systèmes courants de collecte des données, comme les Systèmes d'information pour la gestion de l'éducation (SIGE). En effet, ces 15 dernières années, la plupart des pays ont fait de grandes avancées pour établir des mécanismes pour suivre les progrès accomplis, en particulier vers la réalisation des anciens OMD et plus spécifiquement les objectifs de l'Éducation pour tous (EPT), en mesurant l'accès, la participation et l'achèvement dans l'enseignement primaire formel et l'égalité des genres dans les enseignements primaire, secondaire et supérieur. Dans le programme de I'ODD 4, les pays doivent continuer à collecter ces données tout en améliorant leur qualité pour suivre les progrès accomplis dans le temps.

\section{Politique et fourniture}

Pour suivre pleinement les cibles de l'ODD 4, il est nécessaire de couvrir non seulement les résultats et les produits, mais également les apports aux systèmes éducatifs. Dans l'ensemble, les apports sont les aspects du système éducatif sur lesquels le gouvernement exerce un contrôle relativement direct (comme les dépenses totales, la législation, la structure), tandis que les produits de l'éducation sont les résultats directs de ces apports, et les résultats sont les objectifs ultimes que le système dans son ensemble est censé atteindre, comme les résultats d'apprentissage. Par conséquent, certains indicateurs visent à comparer les pays en relation avec la structure et les ressources fournies aux élèves et aux enseignants, ainsi que le cadre normatif guidant les politiques nationales d'éducation.

\section{Et ensuite...}

La partie suivante de ce guide présente le concept et la définition de chaque indicateur mondial, les méthodes de calcul, les sources de données et les défis méthodologiques. L'annexe présente la liste complète de tous les indicateurs mondiaux et thématiques de l'ODD 4. 
L'indicateur actuel présente les données publiées par chaque organisation spécialisée dans les évaluations transnationales de l'apprentissage. Les données ne sont comparables qu'entre les pays qui ont participé à la même évaluation. Les méthodes pour comparer les résultats de différentes évaluations transnationales de l'apprentissage sont en cours d'élaboration.

\section{MÉTHODE DE CALCUL}

L'indicateur est calculé comme le pourcentage d'enfants et de jeunes du niveau d'enseignement approprié qui atteignent ou dépassent le seuil de compétence prédéfini dans une matière donnée.

\section{INTERPRÉTATION}

Une norme minimale sera établie pour chacun des trois points de mesure. II existe uniquement un seuil qui divise les élèves : (a) inférieur ou (b) égal ou supérieur au SMC. Le seuil inférieur est le pourcentage d'élèves qui n'atteignent pas le SMC tel qu'il est établi par les pays selon les compétences minimales définies à l'échelle mondiale. Le seuil égal ou supérieur est le pourcentage d'élèves qui ont atteint au moins le SMC tel qu'il est défini dans l'évaluation.

Le Document d'information de l'ISU n 48 (2017d) examine les possibilités et les limitations d'élaborer

une stratégie mondiale d'évaluation pour l'Indicateur 4.1.1 en prenant en compte à la fois les aspects

techniques et politiques des évaluations transnationales.

\section{SOURCES DE DONNÉES}

Différentes évaluations transnationales d'apprentissage, notamment : PASEC, PIRLS, PISA, SACMEQ, TERCE et TIMSS.

Le document «Métadonnées relatives aux indicateurs mondiaux et thématiques pour le suivi et l'examen de l'ODD 4 et de l'Éducation 2030" est la principale référence de l'ISU pour des informations sur chaque indicateur de l'ODD 4. Le document sur les métadonnées, actualisé tous les ans, fournit une description détaillée des indicateurs, ainsi que de leurs méthodes de calcul, leurs sources de données et leurs limites (ISU, 2017a).

\section{DÉFIS MÉTHODOLOGIQUES}

II existe trois principaux défis liés à la production d'indicateurs sur les résultats d'apprentissage comparables au plan transnational. Premièrement, les données nationales, régionales et mondiales sur les compétences de base en matière de lecture, d'écriture et de calcul sont collectées fréquemment, mais elles ne peuvent pas être utilisées de manière intégrée pour brosser un tableau mondial de l'apprentissage. Par exemple, les compétences des élèves brésiliens peuvent être comparées à celles des élèves paraguayens car les deux pays ont participé à la même évaluation régionale. Cependant, leurs compétences ne peuvent pas être comparées à celles des élèves sud-africains parce que l'évaluation régionale à laquelle participe l'Afrique du Sud utilise un cadre différent qui est fondé sur des concepts et méthodes différents pour évaluer l'apprentissage.

Deuxièmement, si l'on veut utiliser les données nationales et régionales pour le suivi mondial de l'apprentissage, il est nécessaire d'élaborer des normes techniques communes pour s'assurer que la qualité des données soit similaire. 
Troisièmement, les solutions doivent prendre en compte des points de vue multiples : identifier les domaines pertinents de l'apprentissage qui peuvent et doivent être mesurés à l'échelle mondiale ; conceptualiser comment les données nationales et régionales peuvent contribuer à informer la mesure mondiale ; et enfin, établir un équilibre approprié entre les compétences mondiales et le rôle des influences et les objectifs locaux en matière d'éducation.

L'objectif de l'ISU est d'élaborer des cadres de contenu mondiaux à des fins de référence pour s'assurer que tous les enfants reçoivent l'enseignement dont ils ont besoin, pour améliorer la qualité des données et pour établir des rapports basés sur des métriques communes. Ce travail aidera les gouvernements à mesurer efficacement et à suivre dans le temps les résultats d'apprentissage des élèves en mathématiques et en lecture par rapport à l'Indicateur 4.1.1 de I'ODD et à utiliser les données pour prendre des décisions politiques éclairées. Un autre objectif est de soutenir l'utilisation des évaluations nationales et transnationales existantes pour faciliter la mesure des résultats d'apprentissage et l'établissement des rapports plutôt que de demander à tous les pays d'utiliser une seule évaluation pour l'établissement des rapports sur l'ODD.

Outre les défis ci-dessus mentionnés, il est important de noter une limite importante aux données disponibles. Les évaluations sont administrées d'ordinaire au sein des systèmes scolaires que l'on désigne souvent par évaluations de l'apprentissage organisées à l'échelle de l'école. Les indicateurs actuels ne couvrent que les enfants scolarisés. Par conséquent, les enfants non scolarisés ne sont pas inclus dans cet indicateur. Évaluer les compétences des enfants et des jeunes non scolarisés nécessiterait des enquêtes auprès des ménages.

L'ISU a élaboré un cadre pour guider la conception et la mise en œuvre des évaluations de l'apprentissage pour soutenir la production de mesures des résultats d'apprentissage conformes aux normes techniques de qualité élevée. Principles of Good Practice in Learning Assessment (Principes des bonnes pratiques dans l'évaluation de l'apprentissage) est un bon ouvrage de référence pour la qualité des données sur les résultats d'apprentissage (ISU et ACER, 2017). 
- Physique - un ou deux des faits suivants sont vrais : l'enfant peut ramasser de petits objets facilement et est suffisamment en bonne santé pour jouer ;

- Socio-émotionnel - au moins deux des faits suivants sont vrais : l'enfant s'entend bien avec les autres enfants, il ne donne pas de coups de pied, ne mord pas ou ne frappe pas les autres enfants, et il n'est pas facilement distrait ; et

- Apprentissage - un ou deux des faits suivants sont vrais : l'enfant peut suivre des instructions simples pour faire quelque chose correctement et, quand on lui donne quelque chose à faire, il est capable de le faire de manière autonome.

\section{MÉTHODE DE CALCUL}

Dans I'IDPE de la MICS, l'indicateur est calculé comme le pourcentage des enfants âgés de 36 à 59 mois démontrant les niveaux de développement appropriés à leur âge dans les domaines mesurés. Une définition couramment acceptée de "en bonne voie» utilisant les normes élaborées à l'échelle nationale et régionale pour l'apprentissage et le développement de l'enfant n'a pas encore été élaborée.

\section{INTERPRÉTATION}

Une valeur élevée indique qu'un grand nombre de jeunes enfants sont bien préparés pour commencer l'école primaire dans les domaines de la santé, de l'apprentissage et du bien-être psychosocial.

\section{SOURCES DE DONNÉES}

Des mesures pour refléter les expériences d'apprentissage de la petite enfance ont été utilisées dans de multiples pays sur des échantillons représentatifs comme : I'IDPE de la MICS, le Prototype de l'Afrique de l'Ouest du Bureau régional de l'UNICEF en Afrique de l'Ouest et du Centre (WCARO), le Programme régional des indicateurs pour le développement de l'enfant (PRIDI) en Amérique latine, les Échelles de développement de l'enfant d'Asie de l'Est et du Pacifique, l'Indice de développement des jeunes enfants et l'Indice des capacités humaines précoces. Citons parmi les nouvelles échelles élaborées avec deux ou trois échantillons représentatifs l'Échelle de mesure de la qualité et des acquis d'apprentissage préscolaire (MELQO), et l'Évaluation internationale de l'apprentissage et du développement de la petite enfance (IDELA).

L'UNICEF aide les pays à collecter et à analyser les données afin de combler les lacunes en données pour suivre la situation des enfants et des femmes par le biais de son initiative internationale d'enquête auprès des ménages : les Enquêtes par grappes à indicateurs multiples (MICS). Pour le quatrième cycle de l'initiative MICS (MICS 4), la collecte des données a été élargie pour inclure un IDPE qui vise à mesurer l'état du développement des enfants dans quatre domaines : alphabétisation et calcul ; physique ; développement socio-émotionnel ; et apprentissage.

\section{DÉFIS MÉTHODOLOGIQUES}

D'autres travaux d'élaboration méthodologique seront nécessaires pour s'assurer que la mesure proposée reflète une définition mondialement acceptée de «en bonne voie" qui soit en conformité avec les normes nationales, pertinente pour les enfants du monde entier et qui reflète avec précision «un développement en bonne voie" dans tous les pays. À cet effet, il est nécessaire de créer des schémas normatifs de développement, ce qui n'a pas encore eu lieu dans la plupart des pays. En outre, davantage de pays devront inclure les questions sur la petite enfance dans les enquêtes nationales auprès des ménages ou participer à l'un des projets internationaux générant des données pour cet indicateur. 


\section{Indicateur 4.2.2}

Taux de participation à des activités organisées d'apprentissage (un an avant l'âge officiel de scolarisation dans le primaire), par sexe

\section{CONCEPT}

Cet indicateur mesure l'exposition des enfants à des activités d'apprentissage organisées l'année précédant le commencement de l'école primaire. Un programme d'apprentissage organisé consiste en une série ou une suite cohérente d'activités éducatives conçue dans l'intention d'obtenir des résultats d'apprentissage prédéterminés ou l'accomplissement d'une série de tâches éducatives spécifiques. Les programmes d'éducation de la petite enfance ou d'enseignement primaire sont des exemples de programmes d'apprentissage organisé. L'âge officiel d'entrée au primaire est l'âge auquel les enfants sont obligés de commencer l'enseignement primaire selon la législation ou les politiques nationales.

\section{DÉFINITION}

Le taux de participation à l'apprentissage organisé (un an avant l'âge officiel d'entrée au primaire) est défini comme le pourcentage d'enfants d'un âge donné qui participent à un ou plusieurs programmes d'apprentissage organisé(s), notamment les programmes qui offrent une combinaison d'enseignement et de soins. La participation à l'éducation de la petite enfance et la participation à l'enseignement primaire sont toutes deux incluses. L'âge variera selon les pays en fonction de l'âge officiel d'entrée au primaire.

\section{MÉTHODE DE CALCUL}

Le nombre d'enfants du groupe d'âge concerné qui participent à un programme d'apprentissage organisé est exprimé en pourcentage de la population totale du même âge.

\section{INTERPRÉTATION}

Une valeur élevée de l'indicateur montre un degré élevé de participation à l'apprentissage organisé immédiatement avant l'âge officiel d'entrée au primaire.

\section{SOURCES DE DONNÉES}

L'ISU produit des séries chronologiques basées sur les données des effectifs scolarisés fournis par les ministères de l'Éducation ou les instituts nationaux de la statistique et les estimations de la population produites par la Division de la population des Nations Unies (DPNU). Les données sur les effectifs scolarisés sont collectées à travers l'enquête annuelle de l'ISU sur l'enseignement formel. Les données sont communiquées selon les niveaux d'éducation définis dans la CITE pour garantir la comparabilité internationale des indicateurs en résultant. L'indicateur peut aussi être calculé à partir des enquêtes auprès des ménages et des recensements de la population qui collecte des données sur la fréquentation scolaire de l'éducation de la petite enfance et de l'enseignement primaire par année simple d'âge.

\section{DÉFIS MÉTHODOLOGIQUES}

La participation à des programmes d'apprentissage pendant les premières années n'est pas à temps plein pour de nombreux enfants, ce qui signifie que l'exposition à des environnements d'apprentissage hors de la maison peut varier en intensité. L'indicateur mesure le pourcentage d'enfants qui sont exposés à un apprentissage organisé, mais pas l'intensité du programme, ce qui limite la capacité à tirer des conclusions sur le degré de réalisation de cette cible. Des travaux supplémentaires sont nécessaires pour s'assurer que la définition des programmes d'apprentissage soit cohérente entre les différentes enquêtes et qu'elle soit définie d'une manière facile à comprendre par les répondants à l'enquête, idéalement avec des données complémentaires collectées sur le temps que les enfants passent dans les programmes d'apprentissage. 

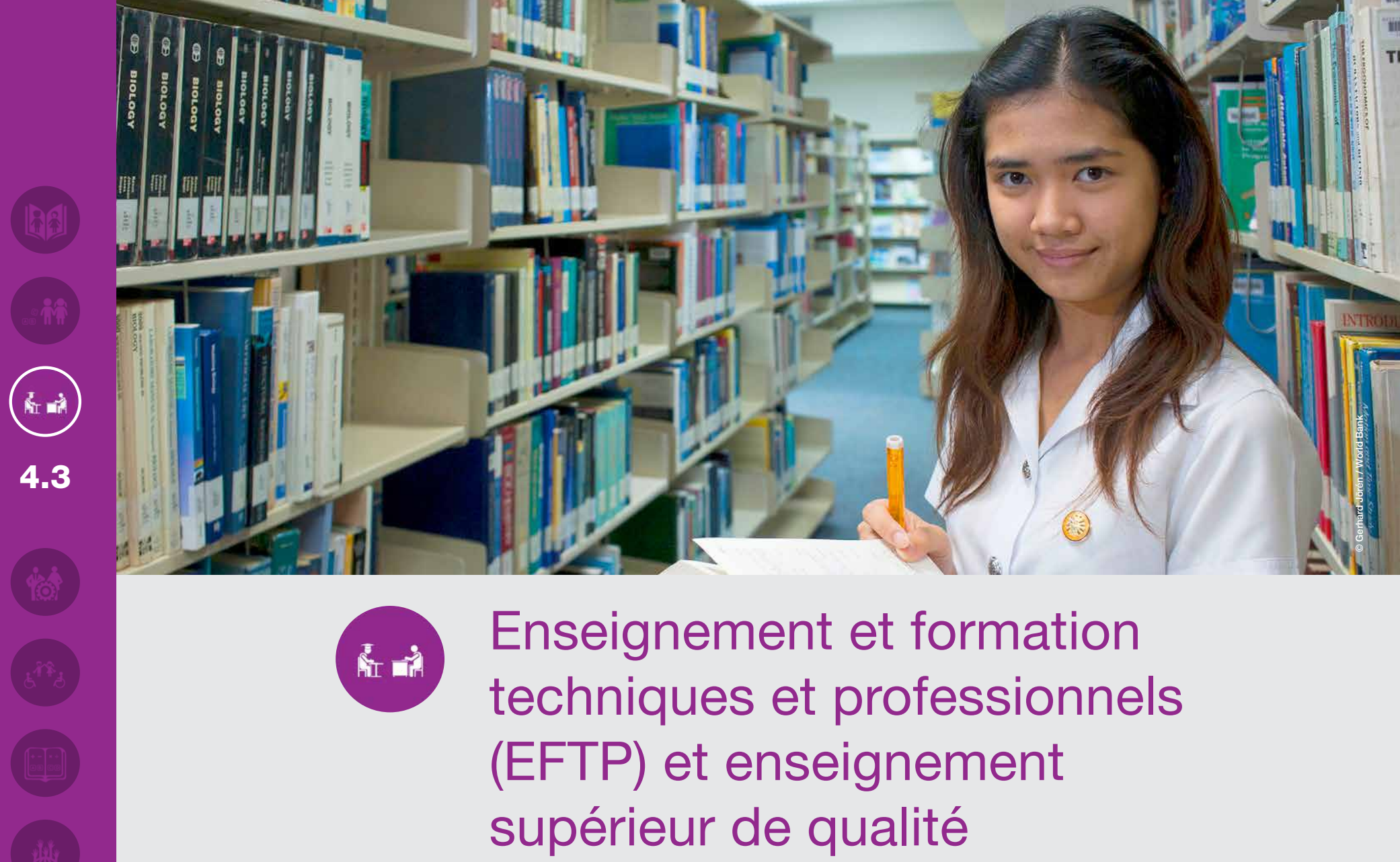

CIBLE 4.3 D'ici à 2030, faire en sorte que les femmes et les hommes aient tous accès dans des conditions d'égalité à un enseignement technique, professionnel ou tertiaire, y compris universitaire, de qualité et d'un coût abordable.

\section{Indicateur 4.3.1}

Pourcentage de jeunes et d'adultes ayant participé à un programme d'éducation et de formation formelle ou non formelle au cours des 12 derniers mois, par sexe

\section{CONCEPT}

La cible couvre plusieurs concepts différents. Bien que l'indicateur mondial ne mesure pas directement la qualité et le coût abordable de l'enseignement, le principal concept "d'accès dans des conditions d'égalité » est évalué en comparant les taux de participation par sexe.

\section{DÉFINITION}

Pourcentage des jeunes et des adultes d'un groupe d'âge donné (par ex. 15-24 ans, 25-64 ans, etc.) participant à un enseignement ou à une formation formels ou non formels pour une période donnée (par ex. au cours des 12 derniers mois). Idéalement, l'indicateur doit être ventilé par type de programme comme l'EFTP, l'enseignement supérieur, l'éducation des adultes et les autres types de programmes concernés, et il couvre à la fois les programmes formels et non formels. Grâce à la CITE 2011, la méthodologie relative aux indicateurs de participation à l'enseignement et à la formation formels est fiable et bien établie dans la plupart des pays. En revanche, les méthodes pour mesurer la participation à l'enseignement et à la formation non formels varient considérablement dans le monde. 


\section{MÉTHODE DE CALCUL}

\begin{tabular}{|l|l|}
\hline+ & $=$ \\
\hline$x$ & $=$ \\
\hline
\end{tabular}

Le nombre de personnes de groupes d'âge sélectionnés participant à un enseignement ou à une formation formels ou non formels est exprimé en pourcentage de la population du même âge.

\section{INTERPRÉTATION}

Une valeur élevée indique qu'une proportion importante de la population appartenant au groupe d'âge concerné participe à un enseignement ou à une formation formels ou non formels.

\section{SOURCES DE DONNÉES}

II existe déjà des méthodologies mises en œuvre au niveau national et international pour collecter les données de l'Indicateur 4.3.1. Au niveau international, les enquêtes comme l'Enquête sur l'éducation des adultes (EEA) en Europe, le Programme pour l'évaluation internationale des compétences des adultes (PIAAC) de l'OCDE et l'Enquête sur la transition vers la vie active (ETVA) du BIT génèrent des informations périodiques et comparables. Au niveau national, les enquêtes auprès des ménages et les enquêtes sur la population active collectent habituellement des données sur la participation aux programmes d'enseignement formels et aussi non formels, bien que la collecte des données sur la participation à ce dernier type de programmes soit beaucoup moins courante.

\section{DÉFIS MÉTHODOLOGIQUES}

L'enseignement et la formation formels et non formels peuvent être offerts dans une variété de cadres incluant les écoles et les universités, les environnements professionnels et d'autres endroits, et ils peuvent avoir des durées variées. Les données administratives ne reflètent souvent que l'offre des cadres formels comme les écoles et les universités. Enfin, les taux de participation ne reflètent pas l'intensité ou la qualité de l'offre, ni les résultats de l'enseignement et de la formation offerts. 


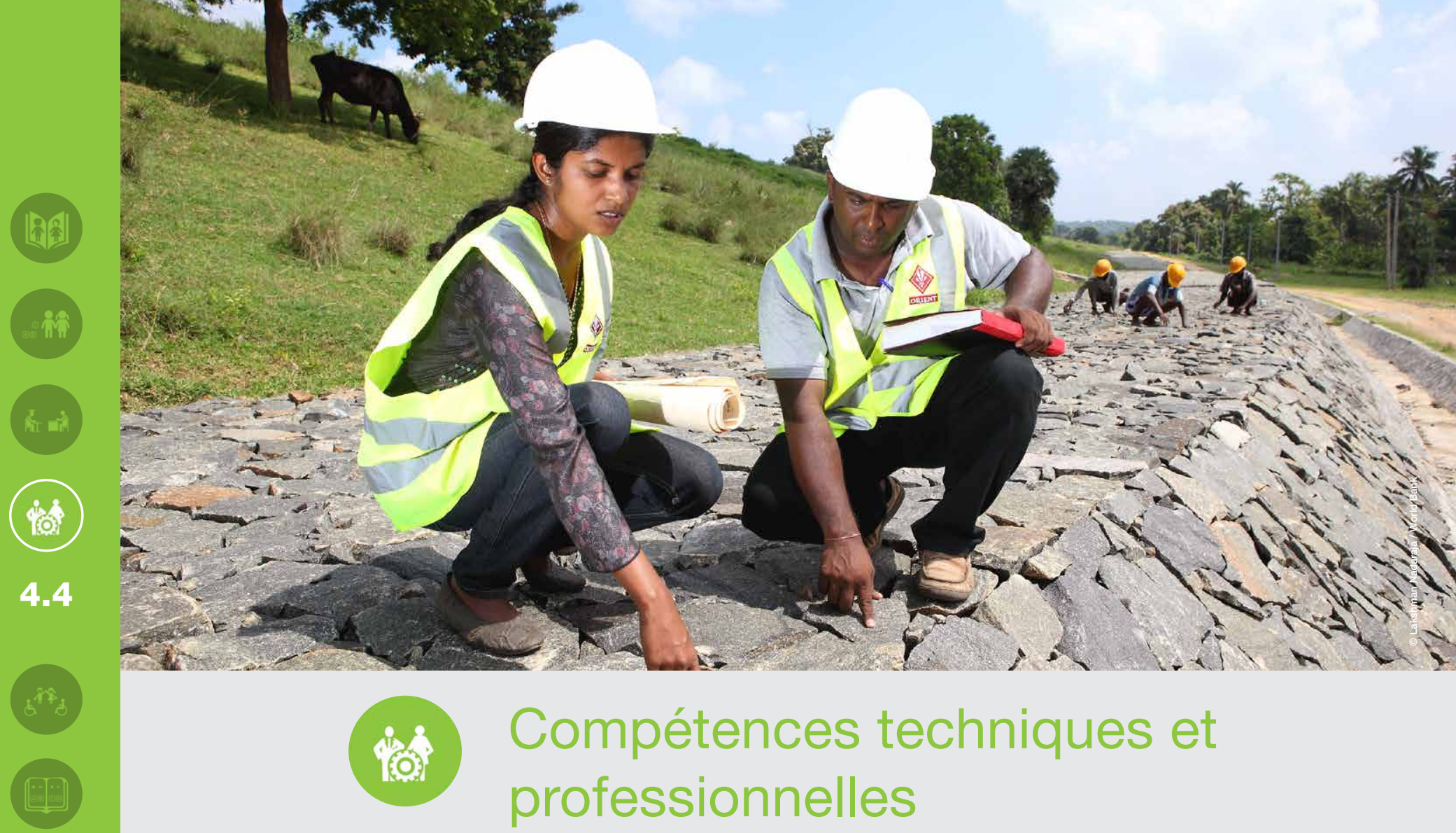

CIBLE 4.4

\begin{abstract}
D'ici à 2030, augmenter considérablement le nombre de jeunes et d'adultes disposant des compétences, notamment techniques et professionnelles, nécessaires à l'emploi, à l'obtention d'un travail décent et à l'entrepreneuriat.
\end{abstract}

\title{
Indicateur 4.4.1
}

Pourcentage de jeunes et d'adultes ayant des compétences en matière de technologies de l'information et de la communication (TIC), par type de compétence

'

Les compétences en matière de TIC déterminent l'utilisation efficace des TIC. L'absence de ces compétences continue d'être l'un des obstacles clés qui empêchent les gens, en particulier les femmes, de bénéficier pleinement du potentiel des TIC. L'indicateur mondial est basé sur le pourcentage d'individus ayant des compétences en matière de TIC par type de compétence. II mesure les compétences en matière de TIC en se basant sur le nombre de personnes qui déclarent avoir suivi des activités liées à l'informatique au cours d'une période donnée (généralement durant les 12 derniers mois dans le cas d'Eurostat ou 3 mois dans le cas de l'Union internationale des télécommunications (UIT)).

DÉFINITION

Pourcentage de jeunes (15 à 24 ans) et d'adultes (15 ans et plus) qui ont entrepris certaines activités liées à l'informatique au cours d'une période donnée (par ex. les trois derniers mois). Les activités liées à l'informatique pour mesurer les compétences en matière de TIC incluent : 
- Copier ou déplacer un fichier ou un dossier

- Utiliser les outils de copier et coller pour dupliquer ou déplacer des informations dans un document

- Envoyer des courriels avec des pièces jointes (par ex. document, image, vidéo)

- Utiliser les formules arithmétiques de base dans une feuille de calcul

- Connecter et installer de nouveaux appareils (par ex. modem, caméra, imprimante)
- Trouver, télécharger, installer et configurer un logiciel

- Créer des présentations électroniques avec un logiciel de présentation (incluant du texte, des images, du son, de la vidéo ou des graphiques)

- Transférer des fichiers entre un ordinateur et d'autres appareils

- Écrire un programme informatique en utilisant un langage de programmation spécialisé

Un ordinateur désigne un ordinateur de bureau, un ordinateur portable ou une tablette (ou un ordinateur de poche similaire). Ne sont pas pris en compte les appareils dotés de certaines fonctions informatisées telles que les postes de télévision ou les téléphones mobiles intelligents.

\section{MÉTHODE DE CALCUL}

L'indicateur est calculé comme le pourcentage de personnes d'une population donnée qui ont répondu «oui » à chacune des compétences en matière de TIC mesurées.

\section{INTERPRÉTATION}

L'indicateur fait le lien entre l'usage des TIC et leur impact, et aide à mesurer et à suivre le niveau de maîtrise des utilisateurs. Une valeur élevée indique qu'une proportion importante de la population de référence a les compétences en matière de TIC mesurées.

\section{SOURCES DE DONNÉES}

La méthodologie a été élaborée par Eurostat et adoptée par I'UIT. Eurostat collecte ces données annuellement pour 32 pays européens, tandis que l'UIT est chargée de définir les normes et de collecter ces informations pour les pays restants.

\section{DÉFIS MÉTHODOLOGIQUES}

L'un des principaux défis de cet indicateur est sa couverture restreinte des "compétences pertinentes" proposées par la cible. En outre, l'indicateur se base sur des informations auto-déclarées. Les personnes interrogées fournissent des informations sur les types d'activités qu'ils ont entrepris mais pas sur leur niveau de compétence. Il est impossible de vérifier l'exactitude de ces auto-évaluations, et plus important encore, il peut exister en matière de déclaration de grandes différences entre des groupes dont les milieux culturels et les situations personnelles sont différents. Par exemple, les femmes sous-estiment souvent dans leurs déclarations leurs aptitudes à utiliser les ordinateurs et Internet, alors que les hommes ont tendance à surestimer les leurs.

Il est également très probable qu'une personne d'un pays donné abordera la question de manière différente qu'une personne d'un autre pays. En ce qui concerne la couverture de la population, la cible des jeunes et des adultes insiste sur le fait que les jeunes devraient être spécifiquement couverts par la mesure. Le contexte est pertinent et peut être très différent d'un pays à l'autre. Les enfants des pays à revenu élevé peuvent développer des compétences des années avant ceux des pays à revenu faible. 


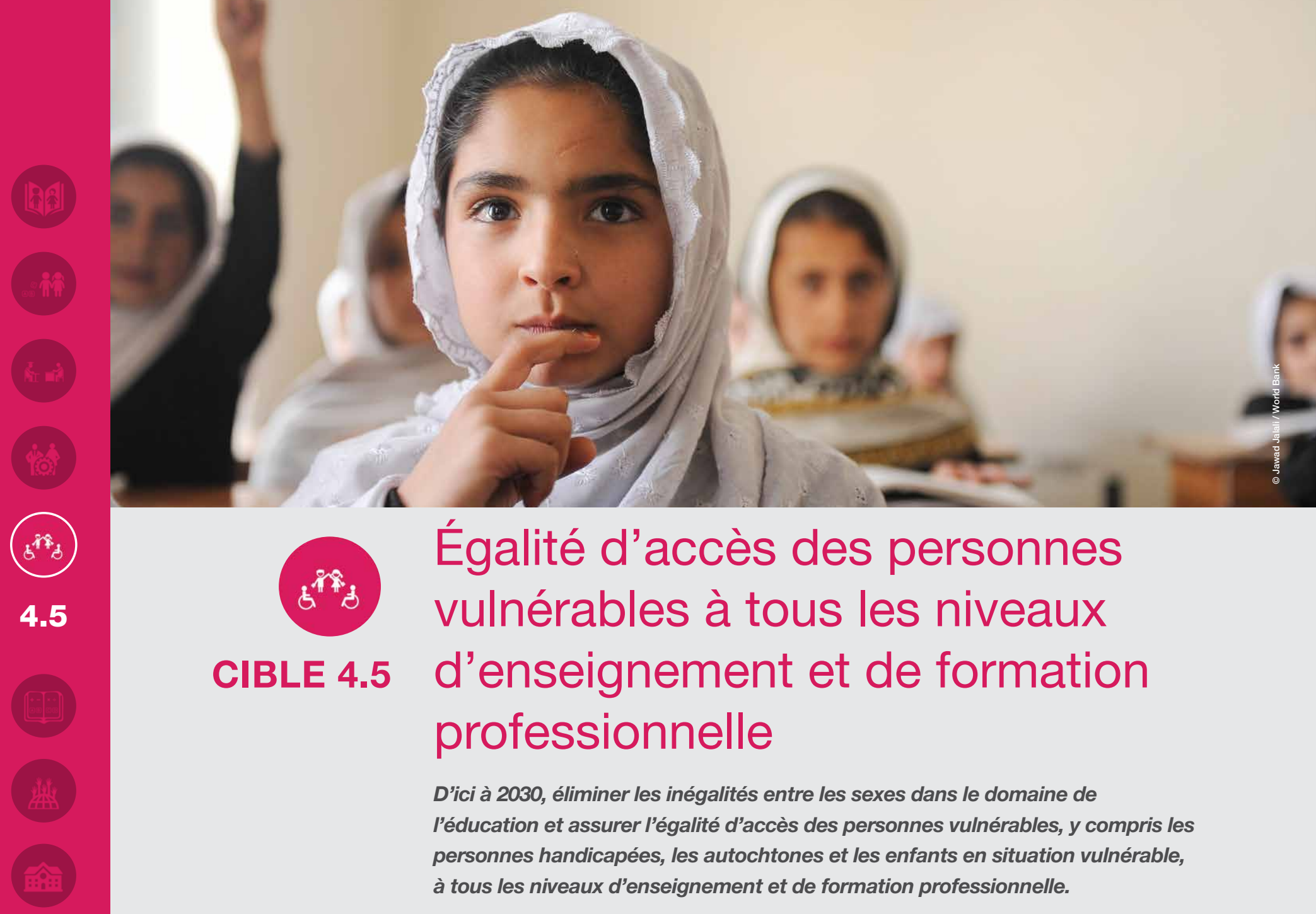

\section{Indicateur 4.5.1}

Indices de parité (femmes/hommes, urbain/rural, quintile inférieur/supérieur de richesse et autres paramètres tels que le handicap, le statut d'autochtone et les situations de conflit, à mesure que les données deviennent disponibles) pour tous les indicateurs de l'éducation de cette liste pouvant être ventilés

\section{CONCEPT}

L'équité est l'une des caractéristiques les plus marquantes du nouveau programme international. De manière générale, ce terme fait référence à différents concepts liés à l'impartialité et aux mesures compensatoires qui reconnaissent le désavantage. L'indice de parité est l'indicateur clé qui sera utilisé pour le suivi mondial entre tous les indicateurs ventilés. En conséquence, les indicateurs liés à l'équité représentent la part la plus importante des données nécessaires pour suivre l'ODD 4 dans son ensemble.

\section{DÉFINITION}

Les indices de parité exigent des données de groupes spécifiques de populations. Ils représentent le ratio de la valeur de l'indicateur d'un groupe par rapport à la valeur d'un autre groupe. D'ordinaire, le groupe le plus susceptible d'être défavorisé est au numérateur. Un indice de parité de 1 exactement signifie que les valeurs de l'indicateur des deux groupes sont identiques, tandis que par convention, les valeurs situées entre 0,97 et 1,03 sont interprétées comme indiquant la parité entre les deux groupes. 


\section{MÉTHODE DE CALCUL}

La valeur de l'indicateur du groupe le plus susceptible d'être défavorisé est divisée par la valeur de l'indicateur de l'autre sous-groupe de population.

\section{INTERPRÉTATION}

Plus l'indice de parité est éloigné de 1, plus la disparité entre les deux groupes de populations est importante.

Tableau 4.5.1 Indicateur 4.1.1b dans le pays A, par sexe

\begin{tabular}{lcc} 
& Filles & Garçons \\
\hline $\begin{array}{l}\text { Nombre d'élèves en dernière année du cycle primaire atteignant ou dépassant } \\
\text { le SMC }\end{array}$ & 25 & 15 \\
Nombre total d'élèves en dernière année du cycle primaire & 50 & 50 \\
Indicateur 4.1.1b & $\mathbf{5 0} \%$ & $\mathbf{3 0} \%$
\end{tabular}

L'indice de parité entre les sexes (IPS) représente le ratio de la valeur de l'indicateur pour les filles par rapport à la valeur des garçons. Par conséquent, l'IPS de l'Indicateur 4.1.1b du pays A résulte de la division de la valeur des filles (50 \%) par la valeur des garçons (30\%) qui est égale à 1,67. Une valeur comprise entre 0,97 et 1,03 reflétera la parité entre les sexes, tandis que les valeurs inférieures à 0,97 indiqueront un avantage pour les garçons et les valeurs supérieures à 1,03 un avantage pour les filles.

\section{SOURCES DE DONNÉES}

0. - Les sources des indices de parité sont précisément les indicateurs calculés pour chacune des autres cibles. Cependant, toutes les données utilisées par les indicateurs mondiaux et thématiques ne permettent pas tous les types de ventilation requis pour le calcul des indices de parité.

\section{DÉFIS MÉTHODOLOGIQUES}

Les efforts pour produire des données et des indicateurs pour le suivi de l'équité dans l'éducation ne doivent pas se faire au détriment de la qualité des données, ni ne doivent être négligés à cause d'un contexte de vulnérabilité ou de ressources insuffisantes. À ce jour, les États membres n'ont pas encore intégré l'équité dans leur suivi national périodique de l'éducation, bien que le nouvel élan insufflé par les ODD engendre la nécessité d'élaborer une stratégie commune et acceptée. Cette approche exigera de parvenir à un consensus sur la définition de l'équité, des métriques et des normes communes ainsi que des mécanismes de coordination pour réduire les coûts de transaction.

\section{Groupe inter-agences sur les indicateurs de l'inégalité dans l'éducation (GIA-IIE)}

En réponse à l'appel d'accroître la priorité accordée à l'équité dans les ODD, I'ISU, I'UNICEF et la Banque mondiale ont créé le Groupe inter-agences sur les indicateurs de l'inégalité dans l'éducation (GIA-IIE). II vise à promouvoir et à coordonner l'utilisation des données provenant des enquêtes auprès des ménages pour le suivi au niveau national, régional et mondial. L'amélioration de la coordination contribuera à garantir l'établissement des rapports normalisés pour compléter les informations disponibles provenant des données administratives, généralement collectées par les systèmes scolaires.

Le GIA-IIE s'inspire en partie de l'expérience de l'Initiative mondiale en faveur des enfants non scolarisés (OOSCI) de I'ISU-UNICEF qui visait à renforcer les données factuelles pour la formulation de politiques nationales d'éducation en combinant des données provenant de sources multiples et en identifiant les caractéristiques des enfants et des adolescents exclus de l'éducation. 

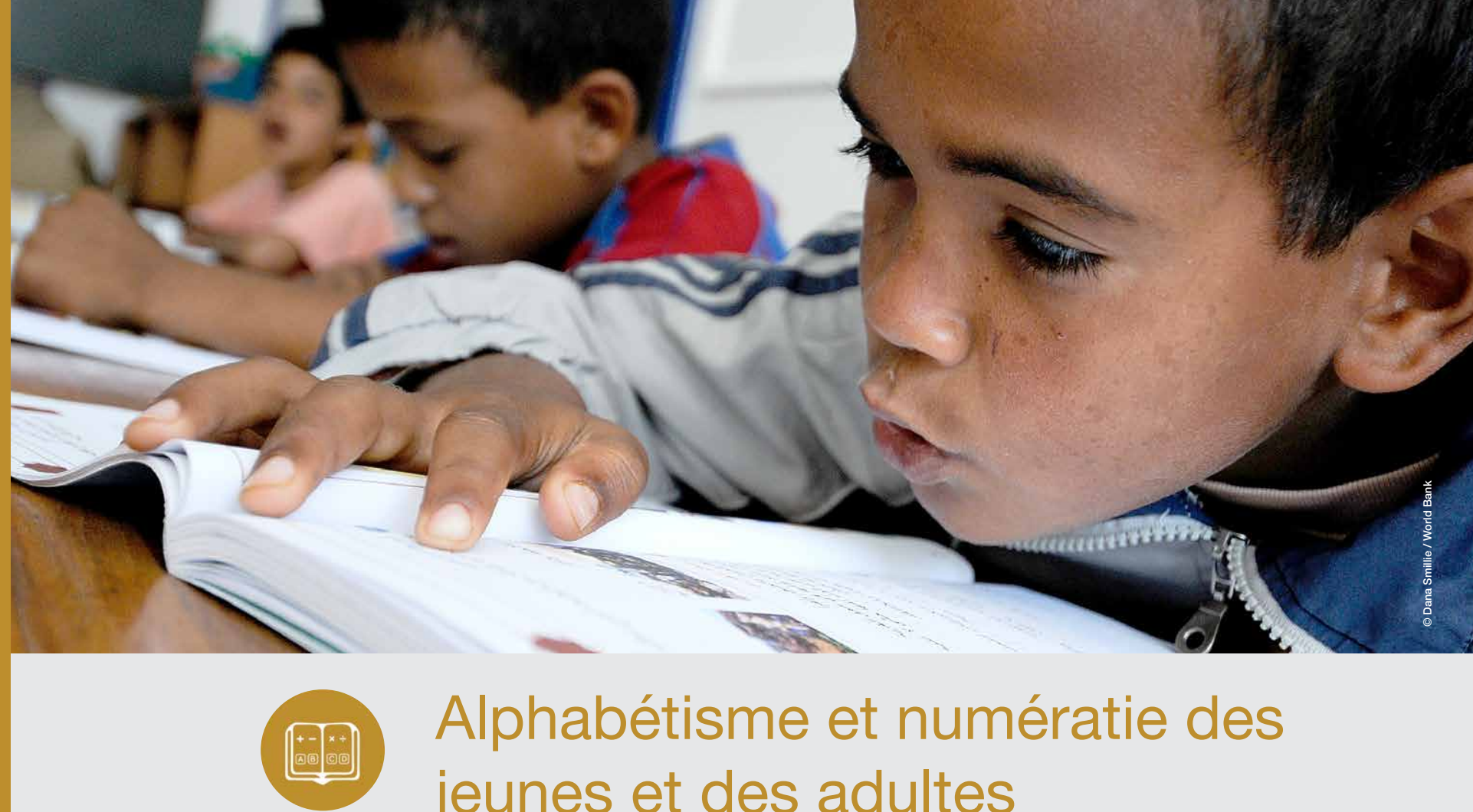

\title{
Alphabétisme et numératie des jeunes et des adultes
}

\section{CIBLE 4.6}

\author{
D'ici à 2030, veiller à ce que tous les jeunes et une proportion considérable \\ d'adultes, hommes et femmes, sachent lire, écrire et compter
}

\section{Indicateur 4.6.1}

Pourcentage de la population d'une tranche d'âge donnée atteignant au moins un certain niveau de maitrise de compétences fonctionnelles en matière (a) de lecture et d'écriture et (b) de calcul, par sexe

\section{CONCEPT}

Les concepts clés à mesurer comprennent les compétences à lire, écrire et compter. Pour des raisons opérationnelles, l'alphabétisme a très souvent été limité à la capacité à lire et à écrire un énoncé simple, y compris quelques compétences élémentaires en arithmétique (numératie). Cependant, cette définition est simpliste et ne couvre pas adéquatement la complexité de ces concepts. L'UNESCO a suggéré plus récemment une définition améliorée de l'alphabétisme : «la capacité à identifier, comprendre, interpréter, créer, communiquer et calculer, à l'aide de matériels imprimés et écrits associés à des contextes variés. L'alphabétisme implique un continuum d'apprentissage pour permettre aux individus d'atteindre leurs objectifs, de développer leurs connaissances et leur potentiel, et de participer pleinement à leur communauté et à la société plus large».

\section{DÉFINITION}

Pourcentage des jeunes (15 à 24 ans) et des adultes (15 ans et plus) qui ont atteint ou dépassé un niveau de compétence donné en (a) alphabétisme et (b) numératie. Le SMC sera mesuré par rapport aux nouvelles échelles communes de compétence en cours d'élaboration.

Le niveau de compétence fixé est la valeur de référence des connaissances de base dans un domaine (lecture, écriture ou calcul) mesurée par les évaluations de l'apprentissage. Actuellement, il n'existe aucune norme commune validée par la communauté internationale ou les pays. Les données actuelles 
proviennent des organismes et des organisations spécialisés dans les évaluations transnationales organisées à l'échelle des ménages sur les jeunes et les adultes.

\section{MÉTHODE DE CALCUL}

Pourcentage de jeunes et d'adultes qui ont atteint au moins le niveau minimal de compétence défini pour les évaluations à grande échelle (échantillon représentatif) sur l'alphabétisme et la numératie des adultes.

\section{INTERPRÉTATION}

II existe uniquement un niveau qui divise les jeunes et les adultes : inférieur au SMC ou égal ou supérieur au SMC. Le seuil inférieur est le pourcentage de jeunes et d'adultes qui n'ont pas atteint le SMC tel qu'il est établi par les pays selon les compétences minimales définies à l'échelle mondiale. Le seuil égal ou supérieur est le pourcentage de jeunes et d'adultes qui ont atteint au moins le SMC.

\section{SOURCES DE DONNÉES}

Les données de cet indicateur sont collectées par le biais des enquêtes d'évaluation des compétences de la population adulte, par ex. PIAAC, Compétences pour l'employabilité et la productivité (STEP), Programme d'évaluation et de suivi de l'alphabétisation (LAMP), et les enquêtes nationales sur l'alphabétisme et la numératie des adultes. Seul PIAAC mesure les deux compétences. STEP et la Courte enquête sur l'alphabétisme (SLS) mesurent uniquement l'alphabétisme. Les enquêtes du PIAAC et de STEP peuvent être placées sur une échelle commune car elles sont liées psychométriquement au niveau de la conception.

\section{DÉFIS MÉTHODOLOGIQUES}

La mesure des compétences des jeunes et des adultes requiert une certaine forme d'évaluation directe. L'utilisation des évaluations organisées à l'échelle des ménages pour mesurer l'alphabétisme et la numératie peut être coûteuse et difficile à administrer, et peut sous-estimer les compétences fonctionnelles dans des domaines qui sont essentiels à la vie quotidienne mais plus difficiles à évaluer par les approches normalisées. II peut en résulter des représentations inexactes de ce que les jeunes et les adultes savent et peuvent faire, en particulier en relation avec les compétences fondamentales qui peuvent beaucoup varier selon les contextes culturels et l'orthographe. 
Cet indicateur tente de mesurer la quantité et la qualité des apports du pays et de déterminer si la qualité de l'offre d'ECM et d'EDD est adéquate pour remplir leur potentiel de transformation. L'indicateur ne se limite pas à «l'existence» ou à «mentionner» I'ECM et l'EDD dans les politiques, les programmes d'étude, la formation des enseignants et les évaluations des élèves.

L'EDD donne les moyens aux apprenants de prendre des décisions avisées et des actions responsables pour l'intégrité environnementale, la viabilité économique et une société juste, pour les générations actuelles et futures, tout en respectant la diversité culturelle. Cela concerne l'apprentissage tout au long de la vie et fait partie intégrante d'une éducation de qualité.

L'ECM cultive le respect d'autrui, en développant le sentiment d'appartenance à une humanité commune et en aidant les apprenants à devenir des citoyens responsables et actifs du monde. L'ECM vise à donner les moyens aux apprenants d'assumer des rôles actifs pour faire face aux défis mondiaux et les résoudre, et devenir des contributeurs proactifs pour un monde pacifique, tolérant, inclusif et sûr.

\section{MÉTHODE DE CALCUL}

Cet indicateur est fondé sur l'évaluation des rapports transmis par les pays à l'UNESCO, décrivant comment ils intègrent l'ECM et l'EDD dans leurs politiques et systèmes éducatifs.

\section{INTERPRÉTATION}

Les progrès pourraient être interprétés selon la priorité et l'accent mis sur la mise en œuvre de l'ECM et de l'EDD dans les politiques, les programmes d'étude, la formation des enseignants et les évaluations des élèves dans le temps, c.-à-d. savoir si l'existence, la fréquence, la priorité et le champ d'application de la mise en œuvre évoluent entre une collecte de données et la suivante.

\section{SOURCES DE DONNÉES}

En référence au mandat de I'UNESCO de suivre la mise en œuvre de la Recommandation de 1974 sur l'éducation pour la compréhension, la coopération et la paix internationales et l'éducation relative aux droits de l'homme et aux libertés fondamentales, un questionnaire est envoyé tous les quatre ans aux États membres de l'UNESCO. C'est un mécanisme établi sur la base duquel les pays présentent systématiquement à l'UNESCO un rapport sur l'état de la mise en œuvre de la Recommandation de 1974 ; le questionnaire couvre presque tous les aspects de l'indicateur proposé, conformément aux recommandations spécifiques. L'UNESCO analyse les résultats de l'enquête et présente le rapport à la Conférence générale sur l'état des pays. En 2016, l'UNESCO a révisé les terminologies et le format du questionnaire pour accroître sa pertinence et sa facilité d'utilisation, ce qui augmentera le taux de réponse.

Les principes directeurs sur les sources, les approches de collecte de données et les expériences liées à ce sujet peuvent être dérivés des travaux mondiaux de suivi et d'évaluation effectués dans le cadre de la Décennie des Nations Unies pour l'éducation en vue du développement durable (DEDD, 2005-2014). Les autres cadres de suivi des droits de l'homme, les examens sectoriels de l'éducation et d'autres études thématiques peuvent également servir de sources supplémentaires pour cet indicateur.

\section{DÉFIS MÉTHODOLOGIQUES}

L'indicateur ne montre pas si les mesures nationales conduisent aux changements souhaités en matière de résultats d'apprentissage et il n'évalue pas directement les résultats d'apprentissage. Cependant, les politiques éducatives, les programmes d'étude, la formation des enseignants et les évaluations des élèves sont des résultats intermédiaires clés de l'engagement national et des efforts menés pour mettre en œuvre efficacement l'ECM et l'EDD et pour fournir un environnement d'apprentissage propice. 


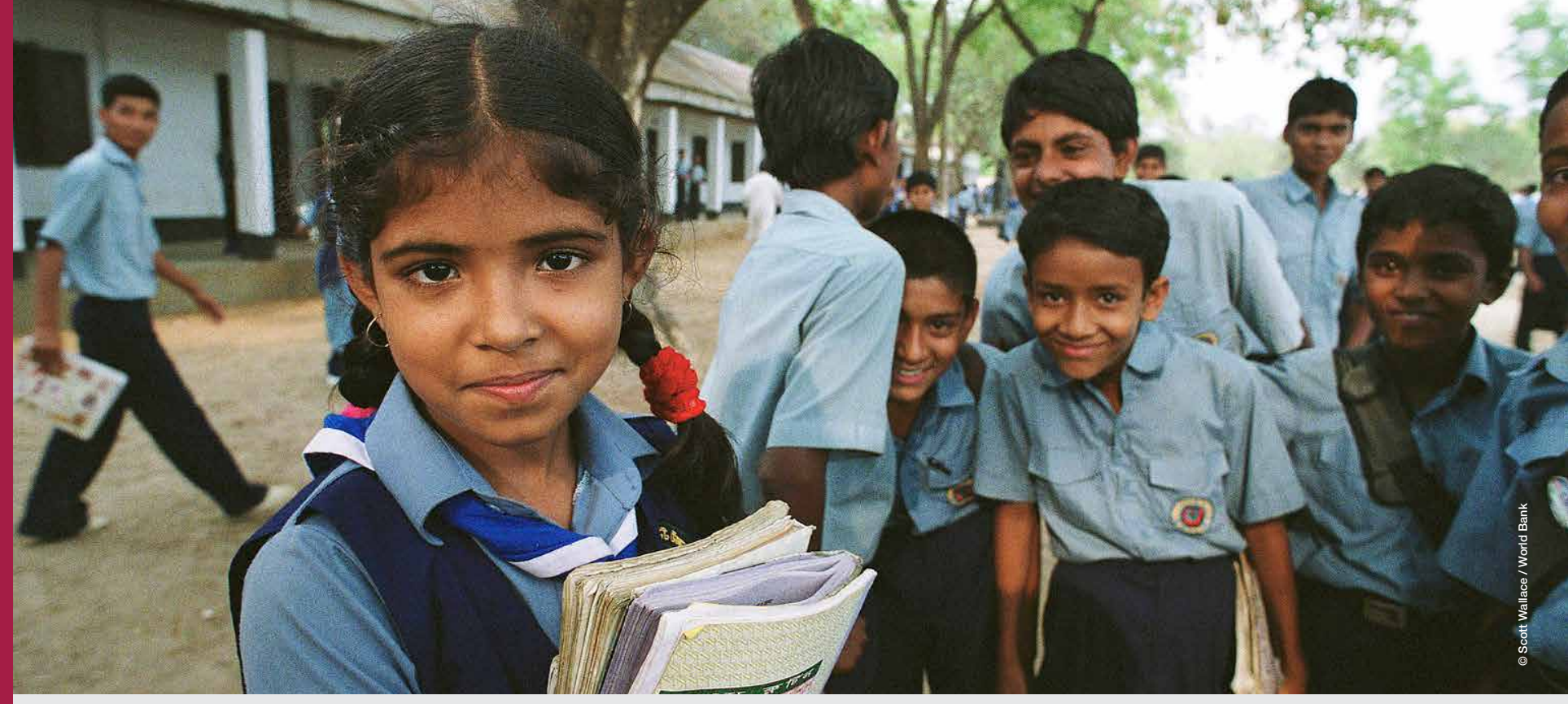

Environnement scolaire

4.a

D'ici à 2030, faire construire des établissements scolaires qui soient adaptés aux enfants, aux personnes handicapées et aux deux sexes ou adapter les établissements existants à cette fin et fournir un cadre d'apprentissage effectif qui soit sûr, exempt de violence et accessible à tous.

Indicateur 4.a.1

Pourcentage d'écoles disposant : (i) de l'électricité ; (ii) de l'Internet à des fins pédagogiques ; (iii) d'ordinateurs à des fins pédagogiques ; (iv) d'infrastructures et de matériels adaptés aux élèves handicapés ; (v) d'un accès élémentaire à l'eau potable ; (vi) d'installations sanitaires de base séparées pour hommes et femmes ; et (vii) d'un minimum de lavabos

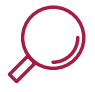

\section{DÉFINITION}

Pourcentage d'écoles par niveau d'enseignement (primaire, premier cycle du secondaire et deuxième cycle du secondaire) qui disposent d'un accès à une installation ou un service donné. Pour les définitions de chaque installation ou service, veuillez consulter : Métadonnées relatives aux indicateurs mondiaux et thématiques pour le suivi et l'examen de l'ODD 4 et de l'Éducation 2030 (ISU, 2017a).

\section{MÉTHODE DE CALCUL}

Le nombre d'écoles à un niveau d'enseignement donné disposant d'un accès aux installations adéquates est exprimé en pourcentage de l'ensemble des écoles de ce niveau d'enseignement.

\section{INTERPRÉTATION}

Une valeur élevée indique que les écoles disposent d'un bon accès aux services et aux installations adéquats. Idéalement, chaque école devrait avoir accès à l'ensemble de ces services et de ces installations.

\section{SOURCES DE DONNÉES}

Données administratives des écoles et des autres prestataires d'enseignement ou de formation. 


\section{Annexe. Cibles et indicateurs de l'Objectif de développement durable 4 sur l'éducation}

Cible 4.1 D'ici à 2030, faire en sorte que toutes les filles et tous les garçons suivent, sur un pied d'égalité, un cycle complet d'enseignement primaire et secondaire gratuit et de qualité, qui débouche sur un apprentissage véritablement utile

4.1.1 Pourcentage d'enfants et de jeunes : (a) en 2e ou 3e année d'études ; (b) en fin de cycle primaire ; et (c) en fin de premier cycle du secondaire qui maîtrisent au moins les normes d'aptitudes minimales en (i) lecture et (ii) mathématiques, par sexe

4.1.2 Organisation d'une évaluation de l'apprentissage représentative à l'échelle nationale (i) en 2e ou 3e année d'études (ii) à la fin du cycle primaire et (iii) à la fin du premier cycle du secondaire

4.1.3 Taux brut d'admission en dernière année du niveau (primaire, premier cycle du secondaire)

4.1.4 Taux d'achèvement (primaire, premier cycle du secondaire et second cycle du secondaire)

4.1.5 Taux d'enfants non scolarisés (cycle primaire, premier cycle du secondaire et second cycle du secondaire)

4.1.6 Pourcentage des enfants ayant dépassé l'âge normal d'un niveau (cycle primaire, premier cycle du secondaire)

4.1.7 Nombre d'années d'enseignement primaire et secondaire (i) gratuit et (ii) obligatoire garanti par le cadre juridique

Cible 4.2 D'ici à 2030, faire en sorte que toutes les filles et tous les garçons aient accès à des activités de développement et de soins de la petite enfance et à une éducation préscolaire de qualité qui les préparent à suivre un enseignement primaire

4.2.1 Pourcentage d'enfants de moins de cinq ans dont le développement est en bonne voie en matière de santé, d'apprentissage et de bien-être psychosocial, par sexe

4.2.2 Taux de participation à des activités organisées d'apprentissage (un an avant l'âge officiel de scolarisation dans le primaire), par sexe

4.2.3 Pourcentage d'enfants de moins de cinq ans vivant dans un environnement d'apprentissage positif et stimulant à la maison

4.2.4 Taux brut de scolarisation dans l'éducation de la petite enfance (a) au préprimaire et (b) dans le développement éducatif de la petite enfance

4.2.5 Nombre d'années d'enseignement préscolaire (i) gratuit et (ii) obligatoire garanti par le cadre juridique

Cible 4.3 D'ici à 2030, faire en sorte que les femmes et les hommes aient tous accès dans des conditions d'égalité à un enseignement technique, professionnel ou tertiaire, y compris universitaire, de qualité et d'un coût abordable

4.3.1 Pourcentage de jeunes et d'adultes ayant participé à un programme d'éducation et de formation formelle ou non formelle au cours des 12 derniers mois, par sexe

4.3.2 Taux brut de scolarisation dans l'enseignement supérieur

4.3.3 Taux de participation aux programmes d'enseignement technique et professionnel (15-24 ans) 
Cible 4.4 D'ici à 2030, augmenter considérablement le nombre de jeunes et d'adultes disposant des compétences, notamment techniques et professionnelles, nécessaires à l'emploi, à l'obtention d'un travail décent et à l'entrepreneuriat

4.4.1 Pourcentage de jeunes et d'adultes ayant des compétences en matière de technologies de l'information et de la communication (TIC), par type de compétence

4.4.2 Pourcentage de jeunes et d'adultes qui ont acquis au moins un niveau minimum de compétences en matière d'alphabétisation numérique

4.4.3 Taux de réussite scolaire des jeunes et des adultes par groupe d'âge, situation au regard de l'activité économique, niveau d'études et orientation du programme

Cible 4.5 D'ici à 2030, éliminer les inégalités entre les sexes dans le domaine de l'éducation et assurer l'égalité d'accès des personnes vulnérables, y compris les personnes handicapées, les autochtones et les enfants en situation vulnérable, à tous les niveaux d'enseignement et de formation professionnelle

4.5.1 Indices de parité (femmes/hommes, urbain/rural, quintile inférieur/supérieur de richesse et autres paramètres tels que le handicap, le statut d'autochtone et les situations de conflit, à mesure que les données deviennent disponibles) pour tous les indicateurs de l'éducation de cette liste pouvant être ventilés

4.5.2 Pourcentage d'élèves de l'enseignement primaire dont la première langue ou la langue maternelle est la langue d'instruction

4.5.3 Mesure dans laquelle les stratégies explicites basées sur une formule réaffectent les ressources de l'éducation aux populations défavorisées

4.5.4 Dépenses d'éducation par élève, par niveau d'enseignement et source de financement

4.5.5 Pourcentage de l'aide totale à l'éducation alloué aux pays à faible revenu

Cible 4.6 D'ici à 2030, veiller à ce que tous les jeunes et une proportion considérable d'adultes, hommes et femmes, sachent lire, écrire et compter

4.6.1 Pourcentage de la population d'une tranche d'âge donnée atteignant au moins un certain niveau de maîtrise de compétences fonctionnelles en matière (a) de lecture et d'écriture et (b) de calcul, par sexe

4.6.2 Taux d'alphabétisation des jeunes et des adultes

4.6.3 Taux de participation des jeunes et des adultes aux programmes d'alphabétisation

Cible 4.7 D'ici à 2030, faire en sorte que tous les élèves acquièrent les connaissances et compétences nécessaires pour promouvoir le développement durable, notamment par l'éducation en faveur du développement et de modes de vie durables, des droits de l'homme, de l'égalité des sexes, de la promotion d'une culture de paix et de non-violence, de la citoyenneté mondiale et de l'appréciation de la diversité culturelle et de la contribution de la culture au développement durable

4.7.1 Mesure dans laquelle (i) l'éducation à la citoyenneté mondiale et (ii) l'éducation en vue du développement durable, y compris l'égalité entre les sexes et les droits de l'homme, sont intégrées à tous les niveaux dans: (a) les politiques nationales d'éducation, (b) les programmes scolaires, (c) la formation des enseignants et (d) l'évaluation des élèves

4.7.2 Pourcentage d'écoles qui dispensent une éducation au VIH et à la sexualité basée sur les compétences utiles dans la vie

4.7.3 Mesure dans laquelle le cadre du Programme mondial d'éducation dans le domaine des droits de l'homme est mis en œuvre à l'échelle nationale (conformément à la résolution 59/113 de l'Assemblée générale des Nations Unies) 
4.7.4 Pourcentage d'élèves par tranche d'âge (ou d'un niveau d'études) montrant une compréhension adéquate des questions relatives à la citoyenneté mondiale et à la durabilité

4.7.5 Pourcentage d'élèves de 15 ans montrant une maîtrise des connaissances de la science environnementale et de la géoscience

Cible 4.a D'ici à 2030, faire construire des établissements scolaires qui soient adaptés aux enfants, aux personnes handicapées et aux deux sexes ou adapter les établissements existants à cette fin et fournir un cadre d'apprentissage effectif qui soit sûr, exempt de violence et accessible à tous

4.a.1 Pourcentage d'écoles disposant : (i) de l'électricité ; (ii) de l'Internet à des fins pédagogiques ; (iii) d'ordinateurs à des fins pédagogiques ; (iv) d'infrastructures et de matériels adaptés aux élèves handicapés ; (v) d'un accès élémentaire à l'eau potable ; (vi) d'installations sanitaires de base séparées pour hommes et femmes; et (vii) d'un minimum de lavabos

4.a.2 Pourcentage d'élèves victimes d'intimidation, de châtiment corporel, de harcèlement, de violence, de discrimination et d'abus sexuels

4.a.3 Nombre d'attaques sur les élèves, le personnel et les établissements

Cible 4.b D'ici à 2020, augmenter considérablement à l'échelle mondiale le nombre de bourses d'études offertes aux pays en développement, en particulier aux pays les moins avancés, aux petits États insulaires en développement et aux pays d'Afrique, pour financer le suivi d'études supérieures, y compris la formation professionnelle, les cursus informatiques, techniques et scientifiques et les études d'ingénieur, dans des pays développés et d'autres pays en développement

4.b.1 Volume de l'aide publique au développement consacrée aux bourses d'études, par secteur et type de formation

4.b.2 Nombre de bourses de l'enseignement supérieur accordées par pays bénéficiaire

Cible 4.c D'ici à 2030, accroître considérablement le nombre d'enseignants qualifiés, notamment au moyen de la coopération internationale pour la formation d'enseignants dans les pays en développement, surtout dans les pays les moins avancés et les petits États insulaires en développement

4.c.1 Pourcentage d'enseignants dans : (a) le préscolaire ; (b) le cycle primaire ; (c) le premier cycle du secondaire ; et (d) le second cycle du secondaire qui ont au moins reçu (avant leur entrée en fonction ou en cours d'activité) les formations minimum organisées pour les enseignants (notamment dans le domaine pédagogique), requises pour l'enseignement à un niveau pertinent dans un pays donné

4.c.2 Ratio élèves/enseignants formés, par niveau d'études

4.c.3 Pourcentage d'enseignants qualifiés selon les normes nationales, par niveau d'études et type d'établissement

4.c.4 Ratio élèves/enseignants qualifiés, par niveau d'études

4.c.5 Salaire moyen des enseignants par rapport aux autres professions exigeant un niveau comparable de formation et de qualification

4.c.6 Taux d'attrition des enseignants par niveau d'études

4.c.7 Pourcentage d'enseignants qui ont bénéficié d'une formation continue au cours des 12 derniers mois, par type de formation

Note : Les indicateurs mondiaux sont présentés sur un fond coloré.

Source : Institut de statistique de l'UNESCO, février 2018. 


\section{Références}

UNESCO (2016). Éducation 2030 : Déclaration d'Incheon et Cadre d'action pour la mise en ceuvre de l'Objectif de développement durable 4. ED-2016/WS/28. Paris : UNESCO. http://unesdoc.unesco.org/Ulis/cgi-bin/ ulis.pl?catno $=245656$

Institut de statistique de l'UNESCO (ISU) (2012). Classification internationale type de l'éducation : CITE 2011. Montréal : ISU. http://uis.unesco.org/sites/default/files/documents/international-standard-classificationof-education-isced-2011-fr.pdf

(2017a). Métadonnées relatives aux indicateurs mondiaux et thématiques pour le suivi et l'examen de l'ODD 4 et de l'Éducation 2030. Montréal : ISU. http://uis.unesco.org/sites/default/files/documents/metadataglobal-thematic-indicators-sdg4-education2030-2017-fr.pdf

(2017b). Rapport 2017 sur les données de l'ODD 4. Le facteur qualité : Renforcer les données nationales afin de surveiller l'Objectif de développement durable 4. Montréal : ISU. http://uis.unesco.org/sites/default/ files/documents/quality-factor-strengthening-national-data-monitor-sdg4-2018-fr.pdf

_ (2017c). "UIS Indicator Development in the Field of Education" (Méthode d'élaboration des indicateurs de l'ISU dans le domaine de l'éducation). Document d'information de l'ISU n 45. Montréal : ISU. http://uis. unesco.org/sites/default/files/documents/ip45-indicator-development-education-methodology-2017-en. pdf

(2017d). "Exploring Commonalities and Differences in Regional and International Assessments" (Explorer les similitudes et les différences dans les évaluations régionales et internationales). Document d'information de l'ISU $n^{\circ}$ 48. Montréal : ISU. http://uis.unesco.org/sites/default/files/documents/ip48-exploring-commonalitiesdifferences-regional-international-assessments-2017-en.pdf

- (2018). "Creating a Global Sharing Network for Strengthening Education Data: edu2030/countrySTAT' (Créer un réseau mondial de partage pour le renforcement des données de l'éducation : edu2030/countrySTAT). Note conceptuelle de I'ISU. Montréal : ISU. http://uis.unesco.org/sites/default/files/documents/creating-globalsharing-network-edu2030countrystat-2018-en.pdf

L'Institut de statistique de l'UNESCO (ISU) et le Centre pour le suivi mondial de l'éducation du Australian Council for Educational Research (ACER) (2017). "Principles of Good Practice in Learning Assessment" (Principes des bonnes pratiques dans l'évaluation de l'apprentissage). http://uis.unesco.org/sites/default/files/documents/ principles-good-practice-learning-assessments-2017-en.pdf

Institut international de planification de l'éducation (IIPE) de I'UNESCO, Institut de statistique de I'UNESCO (ISU) et IIPE-Pôle de Dakar (2016). Méthodologie des comptes nationaux de l'éducation. Paris : UNESCO-IIPE. http:// uis.unesco.org/sites/default/files/documents/methodology-of-national-education-accounts-2016-fr.pdf

Groupe des Nations Unies pour le développement (GNUD) (2017). "Guidelines to Support Country Reporting on the Sustainable Development Goals" (Lignes directrices pour soutenir les rapports des pays sur les objectifs de développement durable). https://undg.org/wp-content/uploads/2017/03/Guidelines-to-Support-CountryReporting-on-SDGs-1.pdf 



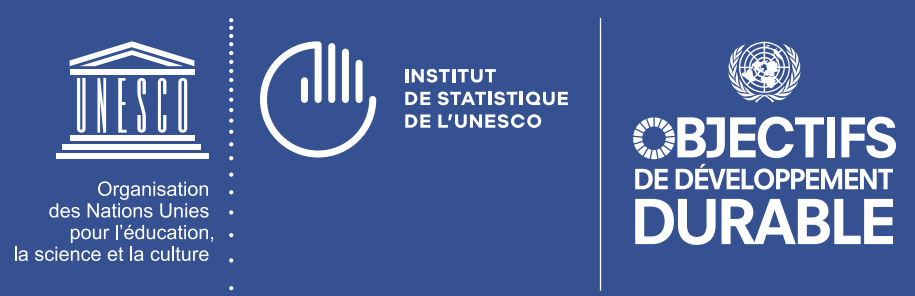

